\title{
Facility Effect Characterization Test of NASA's HERMeS Hall Thruster
}

\author{
Wensheng Huang*, Hani Kamhawi ${ }^{\dagger}$, and Thomas W. Haag ${ }^{\ddagger}$ \\ National Aeronautics and Space Administration Glenn Research Center, Cleveland, OH, 44135, USA \\ and \\ Alejandro Lopez Ortega ${ }^{\S}$ and Ioannis G. Mikellides** \\ Jet Propulsion Laboratory, California Institute of Technology, Pasadena, CA, 91109, USA
}

\begin{abstract}
A test to characterize the effect of varying background pressure on NASA's 12.5-kW Hall Effect Rocket with Magnetic Shielding had being completed. This thruster is the baseline propulsion system for the Solar Electric Propulsion Technology Demonstration Mission (SEP TDM). Potential differences in thruster performance and oscillation characteristics when in ground facilities versus on-orbit are considered a primary risk for the propulsion system of the Asteroid Redirect Robotic Mission, which is a candidate for SEP TDM. The first primary objective of this test was to demonstrate that the tools being developed to predict the zero-background-pressure behavior of the thruster can provide selfconsistent results. The second primary objective of this test was to provide data for refining a physics-based model of the thruster plume that will be used in spacecraft interaction studies. Diagnostics deployed included a thrust stand, Faraday probe, Langmuir probe, retarding potential analyzer, Wien filter spectrometer, and high-speed camera. From the data, a physics-based plume model was refined. Comparisons of empirical data to modeling results are shown.
\end{abstract}

\begin{tabular}{|c|c|c|c|}
\hline \multicolumn{4}{|c|}{ Abbreviations } \\
\hline ARRM & $=$ Asteroid Redirect Robotic Mission & LP & $=$ Langmuir Probe \\
\hline CEX & $=$ Charge-exchange & $\mathrm{MCD}$ & $=$ Mean Channel Diameter \\
\hline FECT & $=$ Facility Effect Characterization Test & RPA & $=$ Retarding Potential Analyzer \\
\hline FP & $=$ Faraday Probe & SEE & $=$ Secondary Electron Emission \\
\hline GRC & $=$ Glenn Research Center & SEP & $=$ Solar Electric Propulsion \\
\hline HERMeS & $\begin{array}{l}=\text { Hall Effect Rocket with Magnetic } \\
\text { Shielding }\end{array}$ & $\begin{array}{l}\text { STMD } \\
\text { TDM }\end{array}$ & $\begin{array}{l}=\text { Space Technology Mission Directorate } \\
=\text { Technology Demonstration Mission }\end{array}$ \\
\hline HSC & $=$ High-Speed Camera & TDU & $=$ Technology Demonstration Unit \\
\hline IPS & $=$ Ion Propulsion System & WFS & $=$ Wien Filter Spectrometer \\
\hline JPL & $=$ Jet Propulsion Laboratory & & \\
\hline
\end{tabular}

\section{Introduction}

$\mathrm{F}^{\circ}$ OR missions beyond low Earth orbit, spacecraft size and mass can be dominated by onboard chemical Fropulsion systems and propellants that may constitute more than 50 percent of the spacecraft mass. This impact can be substantially reduced through the utilization of Solar Electric Propulsion (SEP) due to its substantially higher specific impulse. Studies performed for NASA's Human Exploration and Operations Mission Directorate and

\footnotetext{
*Propulsion Scientist, Electric Propulsion Systems, wensheng.huang@nasa.gov, Senior Member.

${ }^{\dagger}$ Propulsion Scientist, Electric Propulsion Systems, hani.kamhawi-1@ nasa.gov, Associate Fellow.

* Propulsion Scientist, Electric Propulsion Systems, thomas.w.haag@ nasa.gov, Member.

$\S$ Technical Staff, Electric Propulsion Group, alejandro.lopez.ortega@jpl.nasa.gov, Member.

*** Principal Engineer, Electric Propulsion Group, ioannis.g.mikellides@jpl.nasa.gov, Associate Fellow.
} 
Science Mission Directorate have shown that a 50kW-class SEP capability can be enabling for both near term and future architectures and science missions. ${ }^{1}$ A high-power SEP element is integral to the Evolvable Mars Campaign, which presents an approach to establish an affordable evolutionary human exploration architecture. To enable SEP missions at the power levels required for these applications, an in-space demonstration of an operational 50kW-class SEP spacecraft has been proposed as a SEP Technology Demonstration Mission (TDM). In 2010 NASA's Space Technology Mission Directorate (STMD) began developing high-power electric propulsion technologies. ${ }^{2,}{ }^{3}$ The maturation of these critical technologies has made mission concepts utilizing high-power SEP viable.

The Asteroid Redirect Robotic Mission (ARRM) is the leading candidate SEP TDM concept that utilizes an SEP spacecraft to return up to 20 metric tons of asteroidal mass from the surface of a larger asteroid, to a stable orbit around the Moon for subsequent access by a human crewed mission. ${ }^{4-7}$ The Ion Propulsion System (IPS) for ARRM will be used for heliocentric transfer from Earth to the target asteroid, orbit capture at the asteroid, transfer to a low orbit about the asteroid, a planetary defense demonstration after retrieval of the asteroidal mass from the larger asteroid, departure and escape from the asteroid, the heliocentric transfer from the asteroid to lunar orbit, and insertion into a lunar distant retrograde orbit. In addition, the IPS will provide pitch and yaw control of the spacecraft during IPS thrusting. To date, the technology development, performed by the NASA Glenn Research Center (GRC) and the Jet Propulsion Laboratory (JPL), has been focused on an in-house effort to mature the highpower Hall thruster and power processing unit designs.

The high-power Hall thruster is referred to as the Hall Effect Rocket with Magnetic Shielding (HERMeS). In addition to making ARRM viable, the HERMeS propulsion system also has potential commercial applications for raising the orbit of next generation, higher power communication satellites from low-Earth orbit (LEO) to geosynchronous Earth orbit (GEO).

To meet the requirements of the mission concepts under development, the capabilities of the 12.5-kW HERMeS will be enhanced relative to the current state of the art. Characteristics of the thruster include high system efficiency ( $\geq 57 \%$ ), high specific impulse (up to $3000 \mathrm{~s}$ ), and high propellant throughput capability (3400 $\mathrm{kg}$ ). Additionally, HERMeS was designed to deliver similar system efficiency at a more modest specific impulse of 2000 seconds. High specific impulse operation supports mission concepts with high total-impulse requirements like ARRM, while the modest specific impulse operation is beneficial for time-critical operations like LEO to GEO orbit raising.

To verify that the 12.5-kW HERMeS meets the established requirements and to reduce several key risks associated with the thruster, a series of tests are being performed on two Technology Development Units (TDUs). The testing methodology for many of these tests will form the basis for the acceptance and qualification tests of the flight version of the thruster. Figure 1 shows a diagram of the testing on the HERMeS TDUs thus far as well as tests that are planned. Testing that has been completed include the propellant uniformity test ${ }^{8}$, magnetic shielding characterization test ${ }^{9}$, performance characterization test $(\mathrm{PCT})^{10}$, thermal characterization test (TCT $)^{11}$, facility effect characterization test $(\mathrm{FECT})^{10}$, and electrical configuration characterization test $(\mathrm{ECCT})^{12}$. The PCT, TCT, and FECT were performed with a single test setup.

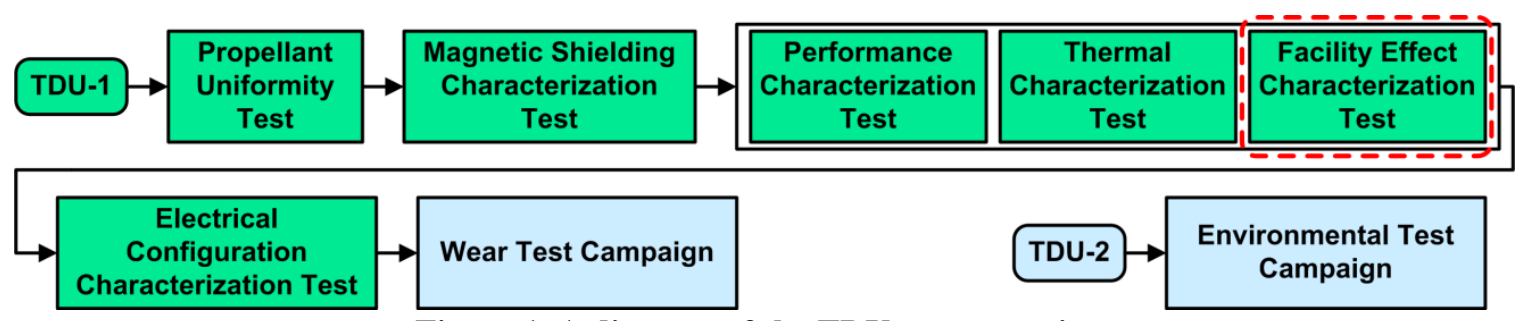

Figure 1. A diagram of the TDU test campaign.

This paper will focus on the FECT that was performed on the TDU1 at NASA GRC. The FECT was partially described in a prior publication. ${ }^{10}$ Following the recent deployments of medium power (1-10 kW) Hall thrusters on orbit, the community has developed renewed interest in the differences in the performance of Hall thrusters in ground facilities versus on-orbit. ${ }^{13-15}$ To reduce the risk that the on-orbit behavior of the HERMeS cannot be predicted from ground test data, the team designed tests to characterize the finite background pressure effect, electrical configuration effect, and the effect of sputter-deposition on the operation of the HERMeS. The FECT was devised to characterize the effect of finite background pressure. Tests to characterize the other two of the three aforementioned ground test effects will be described in separate publications. ${ }^{12,16}$

The first primary objective of the FECT was to demonstrate that the empirical tools being developed to predict the zero-background-pressure behavior of the thruster can provide self-consistent results. Although these tools 
cannot be fully verified without on-orbit data, self-consistency provides a useful check to ensure the team's current understanding of the background pressure effect is properly captured by said tools. This is accomplished by characterizing the performance and oscillation properties of the thruster as functions of facility background pressure, applying a combination of analytical and modeling techniques to predict zero-background-pressure characteristics, and checking for consistency. The second primary objective of the FECT was to supply plasma data for spacecraft interaction studies, including spacecraft erosion and solar array interaction studies. The remainder of the paper describes the test setup, results, modeling and analyses, and

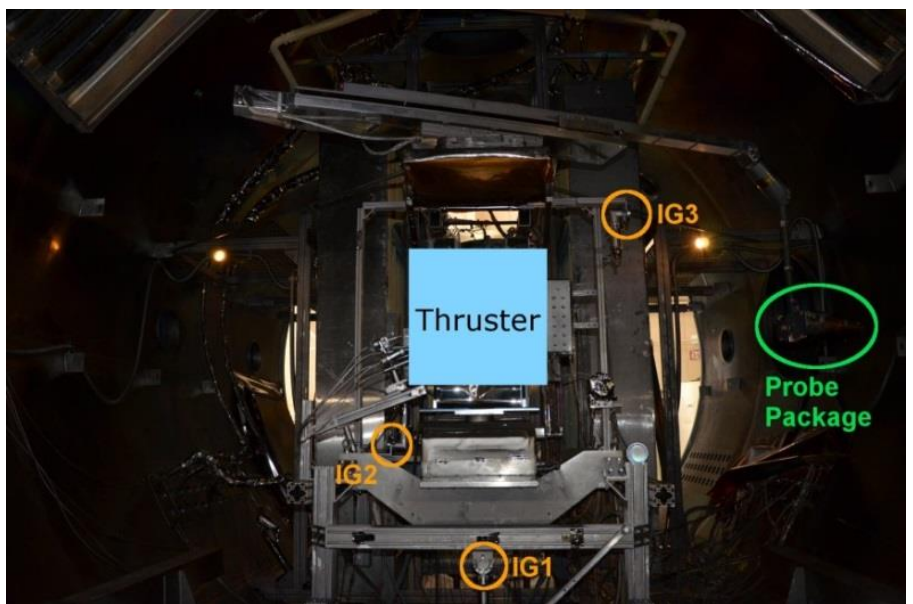

Figure 2. NASA HERMeS TDU1 and thrust stand setup. conclusions of the FECT.

\section{Experimental Setup}

To simplify plot labeling, operating conditions are labeled as vvv-k.k-Pnx, where vvv is the discharge voltage in volts, $\mathrm{k} . \mathrm{k}$ is the discharge power in kilowatts, and $\mathrm{n}$ is the normalized background pressure, defined as the number of multiples of the lowest achievable operating background pressure for the throttle point vvv-k.k. For example, P5x indicates the background pressure is five times that of the lowest achieved.

Unless otherwise noted, all spatial positions presented in this paper have been normalized by the mean channel diameter (MCD) of the thruster. MCD is defined as the average of the inner and outer discharge-channel wall diameters. Furthermore, thruster-centric polar axis $\left(\theta\right.$ axis) is defined as $0^{\circ}$ when viewing directly downstream from the thruster, is negative to the left of the thruster, and is positive to the right of the thruster. Thruster-centric azimuthal coordinate, $\phi$, was defined as $0^{\circ}$ at the 12 o'clock position (vertically the highest point of the discharge channel). The azimuthal angle increases in the clock-wise direction when viewing the thruster from a downstream position. For example, an azimuthal angle of $90^{\circ}$ corresponded to the 3 o'clock position.

\section{A. Thrusters and Test Matrix}

The HERMeS TDU1 was designed to be a $12.5 \mathrm{~kW}, 3000 \mathrm{~s}$, magnetically-shielded Hall thruster. The thruster had been operated over discharge voltages ranging from 300 to $800 \mathrm{~V}$, corresponding to a specific impulse range of 2000 to $3000 \mathrm{~s}$ at full power. The thruster had also been power throttled over discharge powers ranging from 0.6 to $12.5 \mathrm{~kW} .{ }^{10}$ The cathode mass flow rate was maintained at $7 \%$ of the anode mass flow rate.

Thruster magnet coils were energized so that the magnetic shielding topology was always maintained. The only degree of freedom in the magnetic field setting was the strength of the magnetic field. Peak radial magnetic field strength along the discharge channel centerline was chosen as the reference when referring to the strength of the magnetic field. Magnetic field strength was set to provide the best thruster efficiency while having a reasonable amount of margin against oscillation mode transitions. This magnetic field optimization was performed at the lowest achievable background pressure for each throttle point. Figure 2 shows a picture of the NASA HERMeS TDU1 with various test equipment.

For the purpose of FECT, eight thruster throttling points were selected. These throttle points are listed in Table 1. At each throttling point, the thruster settings were optimized at the lowest achievable background pressure. Then, the background pressure was raised by injecting xenon via an

\begin{tabular}{|c|c|c|}
\hline Label & $\begin{array}{c}\text { Discharge } \\
\text { voltage, } \\
\text { V }\end{array}$ & $\begin{array}{c}\text { Discharge } \\
\text { power, } \\
\mathrm{kW}\end{array}$ \\
\hline 300-4.7 & 300 & 4.7 \\
\hline $300-9.4$ & 300 & 9.4 \\
\hline $400-12.5$ & 400 & 12.5 \\
\hline $500-12.5$ & 500 & 12.5 \\
\hline $600-12.5$ & 600 & 12.5 \\
\hline $700-12.5$ & 700 & 12.5 \\
\hline 800-9.7 & 800 & 9.7 \\
\hline $800-12.5$ & 800 & 12.5 \\
\hline
\end{tabular}
auxiliary flow line that exited at $>4$ meters downstream of the thruster pointed away from the thruster. Mass flow rates for the thruster were then adjusted to maintain the same discharge power as background pressure varied. 


\section{B. Test Facility}

Testing was performed in Vacuum Facility 5 at NASA GRC. This cylindrical facility is $4.6 \mathrm{~m}$ in diameter, 18.3 $\mathrm{m}$ long, and was evacuated with a set of cryo-panels. The thruster was mounted on a thrust stand located close to the cryo-panels, with the thruster firing away from the panels. Background pressure near the thruster was monitored with three ion gauges. Figure 2 shows the positions of the three ion gauges relative to the thruster. Ion gauge \#1 points downstream while ion gauges \#2 and \#3 points away from the thruster towards the cylindrical walls of the facility. Correction of gauge readings for effective sensor sensitivity was obtained by calibrating the ion gauges against a spinning rotor gauge in a controlled setup using research grade xenon. Correction of gauge readings for effects of local temperature and direction of the gauge openings relative to the background flux were obtained through a series of modeling studies and verified by experimental data. ${ }^{17}$ The ion gauge studies also showed that pointing the ion gauge opening orthogonal to the firing axis provided the most accurate measurement of the local static pressure. Based on these studies, ion gauges \#2 and \#3 were selected for calculating the local pressure experienced by the thruster. After the aforementioned corrections, the remaining uncertainty in the calculated pressure was dominated by electrical and electronic noise, which was estimated by the manufacturer to be $\pm 6 \%$ of the reading. The lowest pressure achieved for the tested throttle points varied from $3.0 \times 10^{-6}$ to $6.3 \times 10^{-6}$ Torr.

Research-grade xenon propellant was supplied via commercially available mass flow controllers to the thruster, cathode, and auxiliary flow line. These mass flow controllers were calibrated using research-grade xenon prior to testing. Typical uncertainty of measurement was $\pm 1 \%$ of reading.

Electrical power was supplied to the thruster with commercially available power supplies. Separate power supplies supported the main discharge, cathode heater, keeper, inner magnet, and outer magnet. An electrical filter was placed between the thruster and the discharge power supply. All power supplies and the filter were located outside of the vacuum facility.

\section{Time-Averaged Diagnostics}

This section describes the thrust stand and the plasma diagnostics deployed during the FECT. The plasma diagnostics used in this study included a Faraday probe (FP), a Langmuir probe (LP), a retarding potential analyzer (RPA), and a Wien filter spectrometer (WFS). All probes were biased with commercially available power supplies.

The thrust stand used in this study is an inverted pendulum thrust stand designed by Haag. ${ }^{18}$ The thrust stand is actively cooled during operation. For the FECT, the nominal accuracy of the thrust stand was $\pm 1.5 \%$. Periodic invacuum calibration and zero-thrust measurements were made by turn off the thruster and gas flow. Long-term thermal drift was corrected by assuming a linear change in drift between zero-thrust measurements.

Figure 3 shows a photograph of the probe package and the relative position of the four probes in the package. Spatial offset between the probes were accounted for when positioning the probes so that data from different probes can be correlated. Both the RPA and WFS were protected by independent shutters. The probe package was mounted on a boom arm, which was mounted on a set of commercially available motion stages that provided polar and radial motion. Positioning accuracy of this motion system was $<1 \mathrm{~mm}$ for the radial axis and $<0.2^{\circ}$ for the polar axis. The probe package, boom arm, and the bottom of the motion stages were shielded with Grafoil to reduce the amount of backsputtered material.

The FP was of GRC design ${ }^{19,} 20$ and was used to measure ion current density in the farfield plume. Figure 4 shows a cross-sectional diagram of this FP. The collector and guard ring were made of molybdenum and the insulating back was made of Macor. Angular resolution of the FP data was $\sim 0.5^{\circ}$. At each operating condition, the FP was azimuthally swept at five different distances, 4.2, 5.2, 6.1, 7.0, and 7.9 MCD. An additional sweep at 7.9 MCD was conducted to make sure the data was not dependent on the sweep direction. During testing, measurements were made at different bias voltages in increments of $10 \mathrm{~V}$. The results indicated that $-30 \mathrm{~V}$ bias with

respect to facility ground was sufficient to repel incoming electrons for all Figure 3. The probe package. operating conditions.

The LP consisted of a single tungsten wire protruding from an alumina tube. This probe was used to obtain the local plasma potential so that the RPA data can be corrected by this potential. The LP was swept at $3 \mathrm{~Hz}$ for 1 second at each location. FP and LP data were measured by a data acquisition device.

The RPA was of AFRL design. ${ }^{19,}{ }^{20}$ During testing, the electron suppression and repelling grids were biased to $-30 \mathrm{~V}$ with respect to facility ground while the ion retarding grid voltage was swept. The ion retarding grid was biased by a sourcemeter
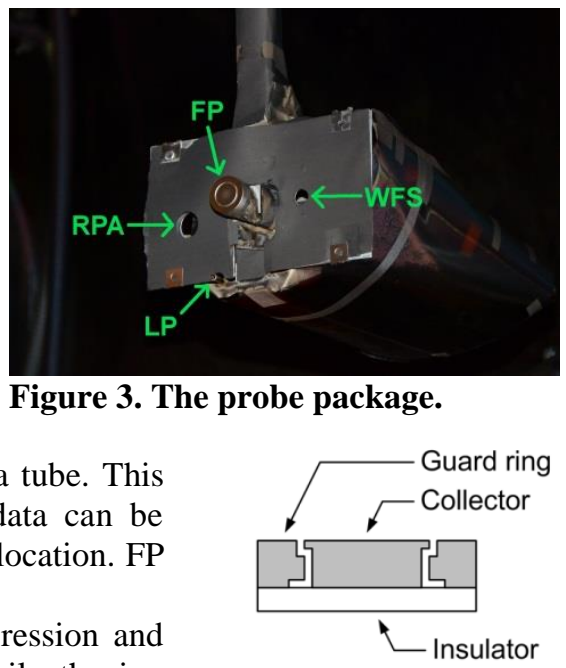

Figure 4. Diagram of the Faraday probe.

4

American Institute of Aeronautics and Astronautics 
while the collected current was measured by a picoammeter. Data were taken at polar angles of $\pm 90^{\circ}, \pm 60^{\circ}, \pm 30^{\circ}$, $\pm 15^{\circ}$, and $0^{\circ}$.

The WFS was a commercial product and was used to measure charged species current fractions. The WFS was the product from a Small Business Innovation Research contract and has a prior history of usage. ${ }^{19-21}$ The electron suppression plate was biased at $-30 \mathrm{~V}$ with respect to facility ground to suppress secondary electron emission (SEE) from the collector. The main bias plate voltage was swept by a picoammeter, which also measured the collector current.

The experiment was conducted via a LabVIEW program from a dedicated data acquisition computer. The computer interfaced with the motion stages via a set of motion controllers. The computer also directly interfaced with the data acquisition device, picoammeters, and sourcemeter. During the experiment, the computer automatically activated the various motion stages, shutters, and probes in the proper sequence.

\section{Time-Resolved Diagnostics}

A high-speed camera (HSC) was deployed to study the oscillation characteristics of the thruster. The HSC was mounted outside of the vacuum facility looking through a window at a mirror inside the vacuum facility. The mirror was a second surface mirror mounted at an angle far downstream of the thruster to reflect light from the thruster to the HSC. The mirror was situated just below the centerline of the vacuum facility to allow an infrared camera mounted downstream of the mirror to have direct line-of-sight to the thruster. Use of the infrared camera was reported in a separate publication. ${ }^{11}$ The mounting structure for the HSC was shielded with Grafoil to reduce the amount of backsputtered material. Figure 5 shows a diagram of the experimental setup inside the vacuum facility.

Three current probes were also installed on the

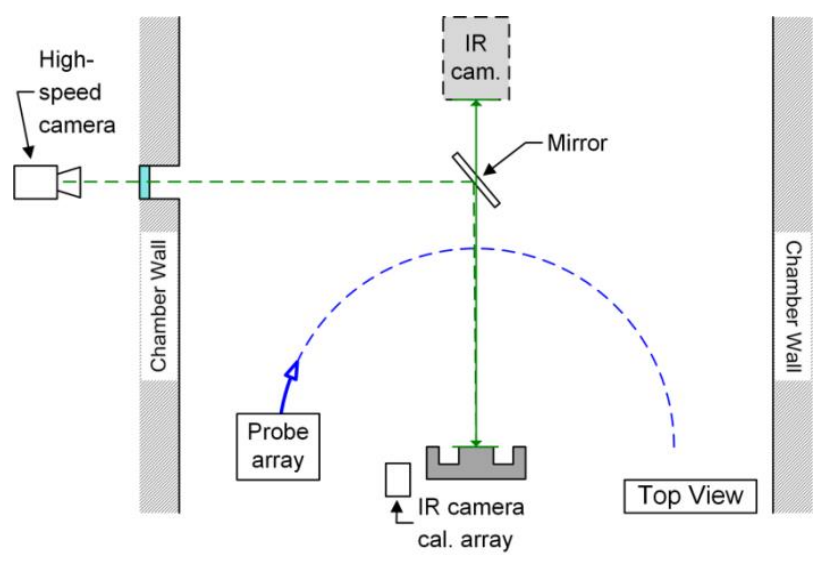

Figure 5. Diagram of the experimental setup inside the vacuum facility. discharge electrical line to provide time-resolved discharge current data. These probes were connected to an oscilloscope, which recorded the data. Current probes were placed both upstream and downstream of the electrical filter on the discharge line. Data from the probe downstream of the electrical filter, which is just upstream of the thruster, were compared to the HSC data. The power spectra from the current probe and the HSC analysis were found to be nearly identical.

\section{Experimental Data Analysis}

\section{A. Phenomenological Model}

A phenomenological model was used to compliment the basic measures of performance to provide a better understanding of the thruster's physical processes. This section describes the equations for thruster efficiency and the phenomenological model. The performance of a Hall thruster can be measured by its anode and total efficiencies, defined in Eqs. (1) and (2), respectively.

$$
\begin{gathered}
\eta_{\mathrm{a}}=\frac{\mathrm{T}^{2}}{2 \dot{\mathrm{m}}_{\mathrm{a}} \mathrm{I}_{\mathrm{d}} \mathrm{V}_{\mathrm{d}}} \\
\eta_{\mathrm{tot}}=\frac{\mathrm{T}^{2}}{2\left(\dot{\mathrm{m}}_{\mathrm{a}}+\dot{\mathrm{m}}_{\mathrm{c}}\right)\left(\mathrm{I}_{\mathrm{d}} \mathrm{V}_{\mathrm{d}}+\mathrm{P}_{\mathrm{m}}+\mathrm{P}_{\mathrm{k}}\right)}
\end{gathered}
$$

In this equation, $T$ is thrust, $\dot{\mathrm{m}}_{\mathrm{a}}$ is anode mass flow rate, $\dot{\mathrm{m}}_{\mathrm{c}}$ is cathode mass flow rate, $\mathrm{I}_{\mathrm{d}}$ is discharge current, $\mathrm{V}_{\mathrm{d}}$ is discharge voltage, $\mathrm{P}_{\mathrm{m}}$ is the electrical power supplied to the magnet, and $\mathrm{P}_{\mathrm{k}}$ is the electrical power supplied to the keeper.

A phenomenological model further breaks the efficiency down into different factors based on physical processes. Many phenomenological efficiency models have been proposed for the Hall thruster in the past. The complexity of these models depended on the operating environment and the state of knowledge in the community at the time. The 
model used in this paper is the same as a prior work by Huang, ${ }^{19}$ which has evolved over time from a number of other studies. ${ }^{21-25}$ The model is shown in Eqs. (3) to (8).

$$
\begin{gathered}
\eta_{\mathrm{a}}=\eta_{\mathrm{v}} \eta_{\mathrm{d}} \eta_{\mathrm{b}} \eta_{\mathrm{m}} \eta_{\mathrm{q}} \\
\eta_{\mathrm{v}}=\frac{\mathrm{V}_{\mathrm{RPA}}}{\mathrm{V}_{\mathrm{d}}} \\
\eta_{\mathrm{d}}=(\cos \delta)^{2} \\
\eta_{\mathrm{b}}=\frac{\mathrm{I}_{\mathrm{b}}}{\mathrm{I}_{\mathrm{d}}} \\
\eta_{\mathrm{m}}=\left(\frac{\mathrm{m}_{\mathrm{Xe}} \mathrm{I}_{\mathrm{b}}}{\dot{\mathrm{m}}_{\mathrm{a}} \mathrm{e}}\right) \alpha_{\mathrm{m}}, \quad \alpha_{\mathrm{m}}=\sum_{\mathrm{k}} \frac{\Omega_{\mathrm{k}}}{\mathrm{Z}_{\mathrm{k}}} \\
\eta_{\mathrm{q}}=\frac{\left(\sum_{\mathrm{k}} \Omega_{\mathrm{k}} / \sqrt{\mathrm{Z}_{\mathrm{k}}}\right)^{2}}{\sum_{\mathrm{k}} \Omega_{\mathrm{k}} / \mathrm{Z}_{\mathrm{k}}}
\end{gathered}
$$

Where $\eta_{\mathrm{a}}$, the anode efficiency, is the same as that calculated from Eq. (1), $\eta_{\mathrm{v}}$ is the voltage utilization efficiency, $\eta_{\mathrm{d}}$ is the divergence efficiency, $\eta_{\mathrm{b}}$ is current utilization efficiency, $\eta_{\mathrm{m}}$ is the mass utilization efficiency, $\eta_{\mathrm{q}}$ is the charge utilization efficiency, $\mathrm{V}_{\mathrm{RPA}}$ is the average ion energy per charge, $\delta$ is the charged-weighted divergence angle, the $\mathrm{I}_{\mathrm{b}}$ is the total beam current, $\mathrm{m}_{\mathrm{Xe}}$ is the mass of a xenon atom, $\mathrm{e}$ is the elementary charge constant, $\alpha_{\mathrm{m}}$ is the part of mass utilization efficiency that depends on charge state information, $\Omega_{\mathrm{k}}$ is the current fraction of the k-th species, and $Z_{\mathrm{k}}$ is the charge of the $\mathrm{k}$-th species.

The voltage utilization efficiency describes how much of the electrical potential provided by the discharge supply is accelerating the ions. This factor was calculated from RPA data. The divergence efficiency describes how much of the kinetic energy imparted to the ions is axial, thrust-producing energy. This factor was calculated from FP data. The current utilization efficiency describes how much of the discharge current is carried by the ions instead of electrons. The electrons generate a negligible amount of thrust compared to ions. This factor was calculated from FP data. The mass utilization efficiency describes how much of the mass flow exiting the thruster is ionized. This factor was calculated from FP and WFS data. The charge utilization efficiency is a number of terms representing the effects of having multiply-charged species that are not already described by the other terms in the efficiency model. This factor was calculated from WFS data.

\section{B. Faraday Probe Analysis}

FP data were used to calculate the plume divergence angle and total ion beam current. The cosine of the momentum-weighted divergence angle is defined as the average axial velocity of the particles divided by the average total velocity of the particles. However, momentum-weighted divergence angle is difficult to measure. The typical approach is to measure the charge-weighted divergence angle, which is approximately equal to the momentum-weighted divergence angle if the multiply-charged current fractions are roughly constant across the interrogated domain. For a polarly-swept probe, Eq. (9) can be used to calculate the charge-weighted divergence angle. For the remainder of the paper, divergence angle refers to the charge-weighted divergence angle.

$$
<\cos \delta>=\frac{2 \pi \mathrm{R}_{\mathrm{FP}}^{2} \int_{0}^{\pi / 2} \mathrm{j}(\theta) \cos \theta \sin \theta \mathrm{d} \theta}{2 \pi \mathrm{R}_{\mathrm{FP}}^{2} \int_{0}^{\pi / 2} \mathrm{j}(\theta) \sin \theta \mathrm{d} \theta}
$$

Where $\theta$ is the polar angle and is equal to $0^{\circ}$ for particles traveling parallel to the firing axis, and $j(\theta)$ is the ion current density as a function of the polar angle. $\mathrm{R}_{\mathrm{FP}}$ is the distance from the Faraday probe collector to the thruster 
center at the exit plane and is constant for a polarly-swept probe. The denominator is equal to the total ion beam current.

A number of effects associated with the use of FP must be accounted for when performing FP analysis. To account for these effects, the FP analysis was divided into four steps. This four-step approach is a natural evolution of FP analysis technique previously developed over many studies. ${ }^{19,20,26,27}$

In step one, the effect of FP gap current was accounted for. For a FP with a guard ring like the one presented in this paper, the effective collection area was not exactly equal to the collector frontal surface area. Current that enters the gap between the collector and the guard ring can be collected by the side surfaces of the collector. ${ }^{27}$ According to work by Brown, the current entering the gap is collected by the collector and the guard ring in a ratio proportional to the ratio of exposed gap area. ${ }^{27}$ For the probe design used in the present study, the area inside the gap was dominated by guard ring surfaces (see Fig. 4). However there is enough area connected to the collector that some level of correction was needed. Only the part of the gap with direct exposure to the incoming ion beam was used in the gap area calculation. Using the approach recommended by Brown, the effective collection area was $\sim 4 \%$ greater than the collector frontal area. The effective collection area was used for all FP analysis.

In step two, the effect of SEE on FP current was corrected. Although the FP was constructed of molybdenum, which is considered a low SEE yield material, some correction for SEE effect was still needed. Secondary electrons born on a negatively biased probe will accelerate away from the probe. This effect adds extra current to the probe measurement that is indistinguishable from the collected ion current. While singly-charged xenon-induced SEE yield for molybdenum is very low, 0.022, the doubly-charged xenon-induced SEE yield is roughly 10 times that of the singly-charged yield, and the triply-charged SEE yield is roughly 35 times that of the singly-charged yield. ${ }^{28-30}$ Since the amount of multiply-charged species in the plume of a Hall thruster is typically not negligible, correction for SEE effect is needed.

Data published by Hagstrum was used to correct for the effect of SEE on the FP measurements. Table 2 summarizes the SEE yield values used in the data analysis of the present study. The singlycharged and doubly-charged xenon-induced yields were averages of the SEE yield data for ion energies in the range of 200 to $800 \mathrm{eV}$ in Hagstrum's 1956 work on molybdenum. ${ }^{29}$ For both of these parameters, the value measured by Hagstrum varied by no more than $10 \%$ of the listed average. Value for the triply-charged xenon-

Table 2. Summary of SEE data for xenon ion bombardment of molybdenum. ${ }^{28,29}$

\begin{tabular}{cc}
$\begin{array}{c}\text { Bombarding } \\
\text { particle }\end{array}$ & $\begin{array}{c}\text { SEE yield of } \\
\text { molybdenum }\end{array}$ \\
\hline $\mathrm{Xe}^{+}$ & 0.022 \\
$\mathrm{Xe}^{2+}$ & 0.20 \\
$\mathrm{Xe}^{3+}$ & 0.70 \\
\hline
\end{tabular}
induced yield of molybdenum could not be found in open literature. The value in Table 2 is an extrapolated value based on the similarity in yield between tungsten and molybdenum. The ratio of triply-charged induced yield to doubly-charged induced yield for tungsten is 3.5 , so the yield for molybdenum is extrapolated to be $3.5 * 0.2$, or 0.7 .

Equation (10) shows the relationship between the actual ion current density and the ion current density measured by the Faraday probe due to SEE effect.

$$
\frac{\mathrm{J}}{\mathrm{J}_{\mathrm{FP}}}=\frac{1}{1+\sum_{\mathrm{k}} \frac{\Omega_{\mathrm{k}} \gamma_{\mathrm{k}}}{\mathrm{Z}_{\mathrm{k}}}}
$$

Where $\mathbf{J}$ is the true ion current density, $\mathbf{J}_{\mathrm{FP}}$ is the current density measured by a nude Faraday probe, and $\gamma_{\mathrm{k}}$ is the SEE yield in number of electrons per ion associated with bombardment by the k-th species. For the present study, the SEE correction factor varied from 0.89 to 0.95 .

Hagstrum also discovered that metastable singly-charged xenon induces roughly the same SEE yield as doublycharged xenon. ${ }^{31}$ In that experiment, Hagstrum varied the energy of the electrons used to generate his singly-charged ion beam from 10 to $70 \mathrm{eV}$. The SEE yield measured increased from 0.022 to 0.025 when the electron energy was ramped up from 25 to $30 \mathrm{eV}$, and then plateaued out at 0.025 up to $70 \mathrm{eV}$. While the Hall thruster ionization zone and an ion beam discharge chamber are not exactly the same, they do share many of the same operating principles, including a reliance on impact bombardment ionization. Since the amount of metastable ions was so small that the SEE yield increased by only 0.003 for Hagstrum's experiment, we assumed the effect was of similar magnitude in a Hall thruster and was negligible.

Ideally, SEE correction would have been calculated as a distribution of polar angle and applied to the FP sweeps. However, past studies have shown that variations in charged species composition with polar angle are relatively small. Data from this study places variation in SEE correction factor with polar angle at $\sim 0.01$. For convenience, a single SEE correction factor was applied for each operating condition. 
Another factor in FP analysis is charge exchange (CEX) effect. However, not all CEX should be removed from FP data because the thruster will generate CEX ions when in space. Therefore, one should not use a gridded FP and reject all CEX ions. Instead, our approach, following recommendations made in Brown's paper, ${ }^{27}$ is to record FP data at multiple distances and multiple pressures.

In step three, two different methods were used to calculate the divergence angle and ion beam current at the exit of the thruster. In the first method, called "global trend", divergence angle and ion beam current were calculated from the FP data at each distance. The angles and currents were then linearly extrapolated to the thruster exit. In the second method, called "source", a zero-distance current density profile was created by linearly extrapolating the data at each polar angle to the thruster exit. The divergence angle and ion beam current of this "source" distribution were calculated and compared to the results from the "global trend" method for consistency.

From re-analysis of a prior study ${ }^{20}$ and from results of this study, the two methods for extrapolating FP data were found to be in disagreement when facility interactions were non-negligible. Plume characteristics tended to trend linearly with background pressure at low pressure and became nonlinear at high pressure. At the same time, disagreement between the two methods for extrapolating FP data greatly increase when the plume characteristics began to trend nonlinearly. Thus, the disagreement between results from the two methods can be used to determine the pressure range for which the FP data analysis is applicable and consistent. Furthermore, re-analysis of a prior study ${ }^{20}$ and current results show that disagreement in calculated divergence angle is more sensitive to background pressure than that in total ion beam current. The disagreement in divergence angle was selected as a key metric for determining consistency.

The final step (step four) in the FP analysis was to predict the characteristics of the thruster at zero background pressure. At each radial and polar position, the current density was linearly extrapolated to zero background pressure. The same set of calculations from step three was then performed on this zero-pressure data set to predict the current density distribution at the exit of the thruster at zero background pressure. As with step three, consistency was checked by using the two methods for calculating divergence angle and ion beam current.

\section{Langmuir Probe and Retard Potential Analyzer Analysis}

LP analysis was carried out using simple Langmuir probe theory. ${ }^{32}$ LP data were ensemble averaged then smoothed using a Savitzky-Golay filter. ${ }^{33,}{ }^{34}$ Plasma potential was set to the probe voltage where the derivative of the probe current with respect to the probe voltage was at its maximum. Uncertainty associated with extracting plasma potential in this manner was $\pm 4 \mathrm{~V}$. This plasma potential was used to correct the RPA reading because the RPA ion retarding grid was biased with respect to the facility ground. The true filter voltage was equal to the ion retarding grid bias voltage minus the local plasma potential.

RPA analysis was carried out by first smoothing the RPA trace using Savitzky-Golay filter, then taking the negative of the derivative of the collector current with respect to the ion retarding grid bias voltage. The result, plotted against the bias voltage, is proportional to the ion energy per charge distribution function. ${ }^{35}$ The average ion energy per charge was calculated by averaging only the part of the trace where the amplitude exceeded half of the maximum amplitude. This averaging approach will be referred to as the threshold-based averaging approach with a $50 \%$ threshold. Figure 6 shows an example of applying the threshold-based averaging approach to an RPA trace. The black dashed vertical line indicates the location of the most probable voltage, the red solid vertical line indicates the result of using the threshold-based averaging approach with the 50\% threshold, and the red dashed horizontal line indicates the $50 \%$ of maximum threshold.

In theory, the most accurate result would have been obtained by ensemble-averaging the entire RPA trace. However, doing so would have produced unphysical results because the ion energy per charge distribution as measured by the RPA was typically much broader than the real distribution due to the wide acceptance angle of the RPA. Using the 50\% threshold-based averaging approach provided a balance between excluding the broadened data and maintaining noise insensitivity. Uncertainty in the average ion energy per charge was $\pm 10 \mathrm{~V}$.

\section{WFS Analysis}

The WFS was used as a velocity filter to separate charged species. Since different charged species in a Hall thruster are accelerated to different velocities, they will show
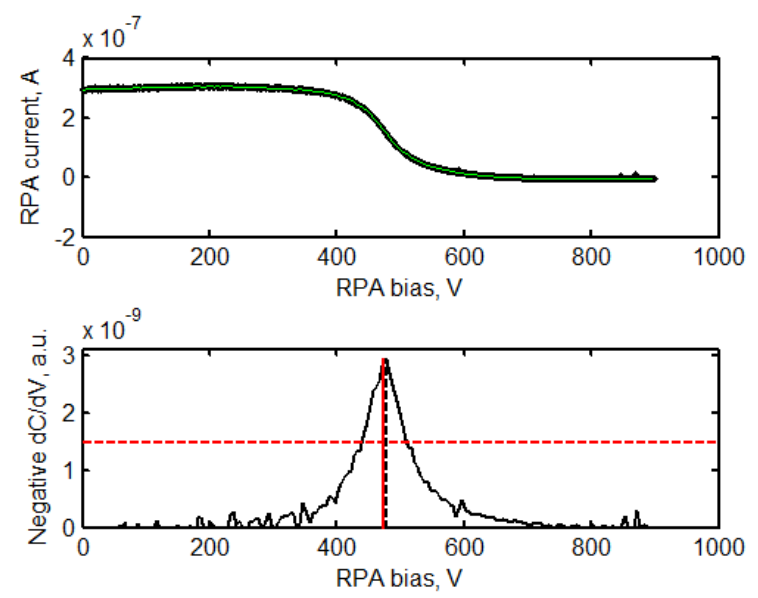

Figure 6. Example RPA analysis plots. 
up as different peaks when interrogated by the WFS. If the WFS velocity resolution is at least several times smaller than the width of the ion velocity distribution function (VDF), the preferred method for analyzing WFS data is via integration. The exact integration method used in this study was thoroughly discussed in a prior publication. ${ }^{36}$ The curve-fit form used was the skew-normal distribution. Corrections for CEX effect were also performed in accordance to prior publications. ${ }^{22,36}$
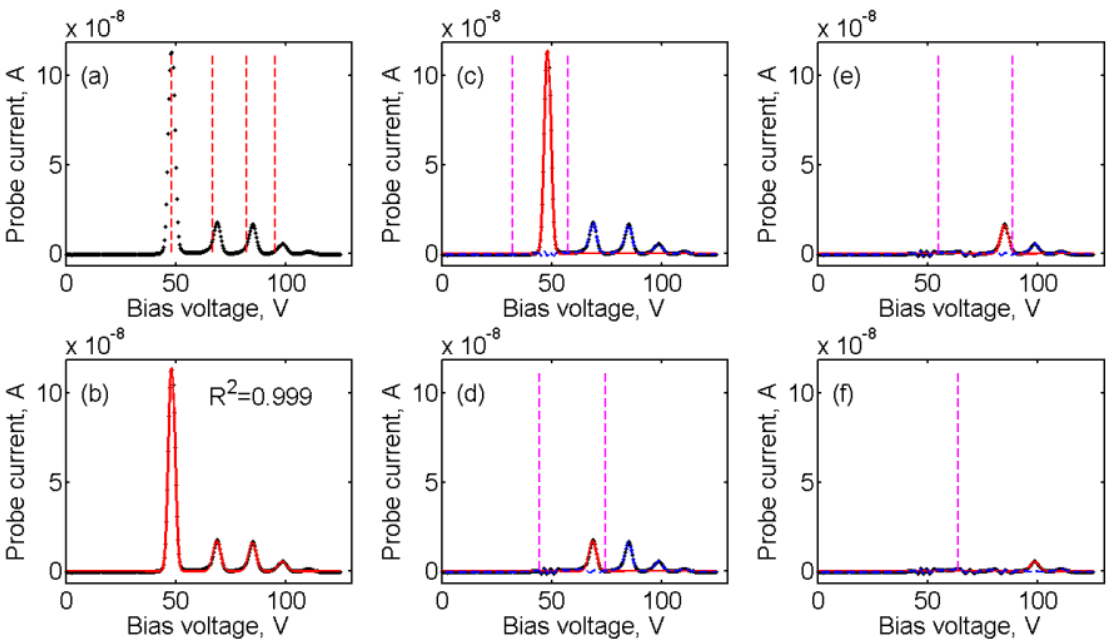

Figure 7 shows an example of Figure 7. Example of analysis plots for Wien filter spectrometer analysis.

analysis program results. For convenience, the subplots are labeled, top to bottom, from left to right, as (a) to (f). Subplot (a) shows the raw WFS data as black data points with red dashed vertical lines showing the approximate location of the first four peaks. Subplot (b) shows the end result from the curve-fitting process in red solid line overlaid on the raw data in black dots. Subplots (c) to (f) show the individual curve-fit steps starting from the $1^{\text {st }}$ peak, then the $2^{\text {nd }}$ peak, and etc. The data prior to the fit at each curve-fit sub-step are shown as black dots, the red solid line shows the curve-fit, the magenta dashed vertical lines show the curve-fit boundaries, and the blue dashed line shows the residual result after subtraction.

\section{E. High-Speed Camera Analysis}

Two types of HSC video were taken. For the thruster HSC videos, the video frame enclosed the entire discharge channel and the frame rate was $180 \mathrm{kfps}$. For the cathode HSC videos, the video frame enclosed only the cathode and the frame rate was $480 \mathrm{kfps}$. The HSC data were processed through a series of steps listed below.

1. The video was averaged over time to create an averaged image.

2. A series of fit were performed on the averaged image to find the center of the thruster and the boundaries of the discharge.

3. The data within the discharge region was divided into 120 azimuthal bins and the average intensity in each bin on each frame was calculated. This binned data was then normalized against the time-averaged values of the binned data. The azimuthal angles of the bins were corrected based on an image calibration performed prior to evacuation of the vacuum facility. Thus, the fact that the HSC image was seen through a mirror situated below the centerline of the vacuum facility was corrected for.

4. A statistical analysis was performed to determine the probability distribution function (PDF) of the intensity.

5. A two-dimensional (2D) Fourier transform was performed to obtain a series of power spectra categorized by the mode number $\mathrm{m}$.

6. The results were plotted against background pressure.

Figure 8 shows an example of a thruster HSC video being analyzed. The left image is the averaged image and the right image shows how the program divided the image up at step three. A dashed green line radiating from the center of the thruster indicates the $\phi=0^{\circ}$ position on the actual thruster. A small green branch perpendicular to the dashed green line points in the actual clock-wise direction. Recall HSC recorded images that were reflected through a mirror. The small red circle indicates a region encompassing the cathode that is excluded from the analysis. Two concentric green circles that sandwich a region highlighted in red indicate the boundaries of the discharge.

The data resulting from analysis step three were the relative fluctuation of each azimuthal bin with time. This step produced results that were much less sensitive to variations in pixel sensitivity, transmission of the viewport, and reflectance of the mirror. Step three also removed any real, time-averaged, azimuthal variation in light intensity. For instance, the presence of a stationary hot spot would be removed by this step. This characteristic was considered acceptable because the present study was focused on transient instead of stationary features. 
For the power spectra obtained from analysis step five, the $\mathrm{m}=0$ (zeroth order) spectrum showed the frequency of any oscillation modes where the entire discharge plasma oscillated together. This phenomenon is referred to as global oscillation. The $\mathrm{m}=1$ (first order) spectrum showed the frequency of any spokes mode with one spoke present, the $m=2$ spectrum showed the frequency of any spokes mode with two spokes present, and so on. ${ }^{13}$ During this study, the main discharge of the HERMeS TDU1 was found to not exhibit any spokes mode behavior at the operating conditions studied. This paper will focus on the global oscillation modes that were present.

Analysis of the global oscillation modes can also be completed by averaging the light intensity data across all bins and performing a 1D Fourier transform. This approach was also performed and the results checked against the $\mathrm{m}=0$ spectrum from 2D Fourier transform. The two approaches were in excellent agreement.

Analysis procedure of the cathode videos was very similar to the analysis of the thruster videos with two important differences. The first difference was that instead of analyzing the

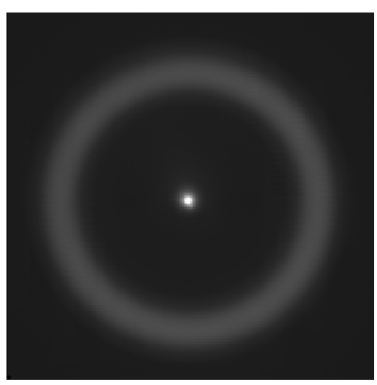

Average image Figure 8. Example of thruster HSC video analysis.

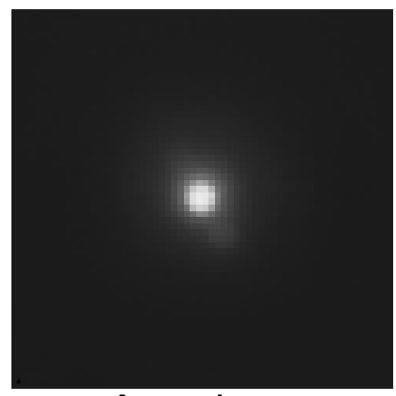

Average image

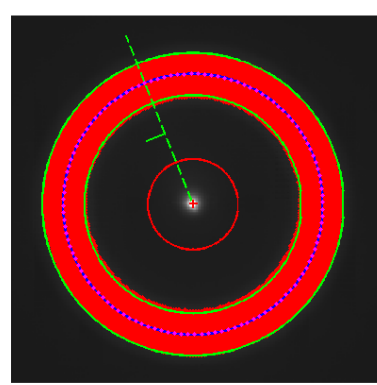

Detection zone

Figure 9. Example of cathode HSC video analysis. discharge channel, cathode HSC analysis focused on the region near the cathode and excluded a very small spot right at the center of the cathode that tended to saturate the HSC sensor. The second difference is that 48 azimuthal bins were used instead of 120 due to the reduction in the number of pixels available per unit azimuthal angle. Figure 9 shows an example of cathode HSC video analysis.

Unlike the thruster videos, the cathode videos exhibited strong $\mathrm{m}=1$ oscillations that corresponds to the gradient-driven mode unique to centrally mounted cathode in a Hall thruster, which were first identified and described by Jorns ${ }^{37}$ For this reason, both $\mathrm{m}=0$ and $\mathrm{m}=1$ power spectra were of note for cathode videos.

\section{Experimental Results}

\section{A. Plume Results for Performance Characterization}

This section will focus on aspects of the test that addresses the first primary objective, which was to demonstrate that the ground test tools for predicting zero pressure thruster and plume behavior are self-consistent. We begin by examining the analysis results from each probe on the probe package from the perspective of performance characterization. The probe results along with thrust stand measurements and basic telemetry will be tabulated to help highlight trends with varying background pressure.

To better understand how the plasma plume changed with background pressure and distance away from the thruster, we begin by examining the FP traces at the $800 \mathrm{~V}, 12.5 \mathrm{~kW}$ throttle point. The plume exhibited similar trends at other throttle points. Figures 10 and 11 show the current density profiles as functions of polar angle at various distances away from the thruster for the 800-12.5-P1X and 800-12.5-P5X operating condition, respectively. Current density is shown in units of Ampere per steradian because evolution in the shape of the profile is readily apparent when current density is plotted this way. From these two figures, one can see that CEX with neutrals (both background and thruster neutrals) have a tendency to raise the current density at the wing $\left(<-45^{\circ}\right.$ and $\left.>45^{\circ}\right)$. This occurred because CEX ions do not carry the momentum of the beam ions and have a large divergence in comparison to the beam ions. Comparing the two figures, one can also see that elevated background pressure led to a large increase in current density at the wing and more prominent evolution in current density with distances. Analysis indicates that the level of CEX activity present in the 800-12.5-P5X case was high enough to invalidate the assumption that the current density profile evolve in a linear fashion. One sign that this assumption was violated was that the extrapolated current density at the exit (the source distribution) has negative current density values at polar angles of $\pm 90^{\circ}$. 


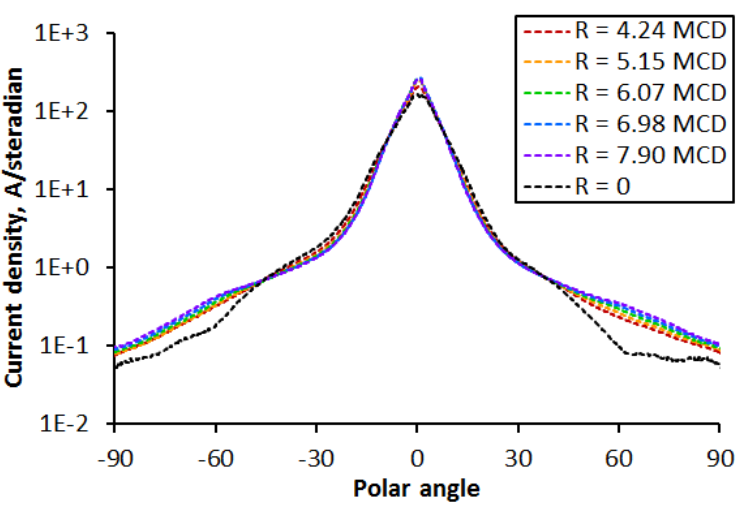

Figure 10. Current density profiles at different distances for the 800-12.5-P1X operating condition.

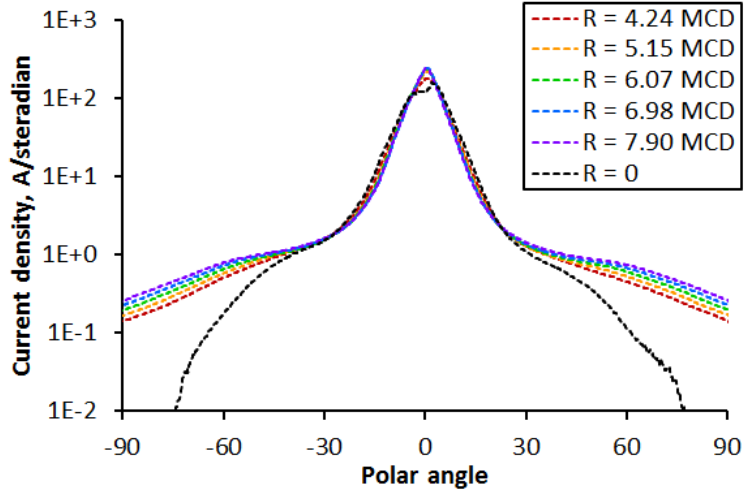

Figure 11. Current density profiles at different distances for the 800-12.5-P5X operating condition.

To find further evidence that the plasma plume structure changed as the background pressure was elevated, the two methods for calculating divergence angle was scrutinized. Tables 3 and 4 show the results from the two methods of calculating divergence angle. Method one is labeled GT for "Global Trend". Method two is labeled S for "Source". The disagreement between the two methods is labeled Dis.

Table 3. Divergence angle in degrees calculated from the "Global Trend" versus "Source" methods for low discharge voltage conditions.

\begin{tabular}{lcccccccccccc}
\hline & \multicolumn{3}{c}{$\mathbf{3 0 0 - 4 . 7}$} & \multicolumn{3}{c}{$\mathbf{3 0 0 - 9 . 4}$} & \multicolumn{3}{c}{$\mathbf{4 0 0 - 1 2 . 5}$} & \multicolumn{3}{c}{$\mathbf{5 0 0 - 1 2 . 5}$} \\
\hline & GT & S & Dis & GT & S & Dis & GT & S & Dis & GT & S & Dis \\
\hline P0X & 23.9 & 23.9 & 0.0 & 26.6 & 26.6 & 0.0 & 25.3 & 25.3 & 0.0 & 20.6 & 20.6 & 0.0 \\
P1X & 22.8 & 22.7 & 0.1 & 25.1 & 25.0 & 0.1 & 23.5 & 23.4 & 0.1 & 19.7 & 19.5 & 0.2 \\
P1.5X & 22.3 & 22.1 & 0.2 & 24.2 & 23.9 & 0.3 & 22.3 & 21.9 & 0.4 & 19.6 & 19.2 & 0.4 \\
P2X & 22.0 & 21.6 & 0.4 & 23.7 & 23.3 & 0.4 & 21.8 & 21.3 & 0.5 & 19.2 & 18.5 & 0.7 \\
P3X & 21.5 & 20.9 & 0.6 & 23.1 & 22.5 & 0.6 & 21.0 & 20.6 & 0.4 & 19.0 & 18.1 & 0.9 \\
P5X $^{\star}$ & 21.0 & 20.2 & 0.8 & & & & & & & 18.9 & 17.8 & 1.1 \\
\hline
\end{tabular}

*GT = Global Trend method, $\mathrm{S}=$ Source method, Dis = disagreement between the two methods. The data in cells corresponding to 500-12.5 and P5X was from the condition 500-12.5-P4.5X.

Table 4. Divergence angle in degrees calculated from the "global trend" versus "source" methods for high discharge voltage conditions.

\begin{tabular}{lcccccccccccc}
\hline & \multicolumn{3}{c}{$\mathbf{6 0 0 - 1 2 . 5}$} & \multicolumn{3}{c}{$\mathbf{7 0 0 - 1 2 . 5}$} & \multicolumn{3}{c}{$\mathbf{8 0 0 - 9 . 7}$} & \multicolumn{3}{c}{$\mathbf{8 0 0 - 1 2 . 5}$} \\
\hline & GT & S & Dis & GT & S & Dis & GT & S & Dis & GT & S & Dis \\
\hline P0X & 20.1 & 20.1 & 0.0 & 20.7 & 20.7 & 0.0 & 25.1 & 25.1 & 0.0 & 20.4 & 20.4 & 0.0 \\
P1X & 19.4 & 19.2 & 0.2 & 19.8 & 19.6 & 0.2 & 24.9 & 24.9 & 0.0 & 19.8 & 19.7 & 0.1 \\
P1.5X & 19.1 & 18.7 & 0.4 & 19.2 & 18.8 & 0.4 & 25.0 & 24.9 & 0.1 & 19.6 & 19.3 & 0.3 \\
P2X & 18.9 & 18.2 & 0.7 & 19.1 & 18.4 & 0.7 & 24.9 & 24.7 & 0.2 & 19.5 & 19.0 & 0.5 \\
P3X & 18.6 & 17.5 & 1.1 & 19.0 & 17.8 & 1.2 & 25.0 & 24.5 & 0.5 & 19.5 & 18.5 & 1.0 \\
P5X $^{*}$ & 18.7 & 16.4 & 2.3 & 19.3 & 17.2 & 2.1 & 25.9 & 25.1 & 0.8 & 19.8 & 17.9 & 1.9 \\
\hline
\end{tabular}

*GT = Global Trend method, $\mathrm{S}=$ Source method, Dis = disagreement between the two methods. The data in cells corresponding to 600-12.5 and P5X was from the condition 600-12.5-P4.5X.

From Tables 3 and 4, one can see that the disagreement in divergence angle between the two methods were relative small but grows quickly with background pressure. At the highest tested operating conditions the differences became pronounced. Since disagreement in the calculated divergence angle between the two methods implied the plume was evolving non-linearly and since non-linear behavior implies non-negligible facility effects, operation at high background pressure should be avoided. Also of interest was the fact that even at the lowest achieved background pressure, where disagreement between the two methods was negligible, the difference between the calculated divergence angle and the predicted zero-pressure divergence angle was not negligible. Here, negligible is defined as $<0.5^{\circ}$, which is the angular resolution of the FP. 
Observation that the evolution of the plasma plume structure became non-linear at elevated background pressure was used as the basis for determining when the FP analysis approach was applicable. To establish a quantifiable metric for the background pressure, we tentatively tried the criterion that the calculated divergence angle must be within $1^{\circ}$ of the extrapolated zero-pressure divergence angle. Figure 12 show a plot of the difference in divergence angle from the zeropressure divergence angle as a function of the background pressure near the thruster. The divergence angle shown in this figure was calculated using the "Source" method since the "Source" method had been shown to be more sensitive to changes in the plume structure.

Figure 12 shows another sign that the evolution of the plume with distance changed from linear to nonlinear as the background pressure rose. With the exception of the 800-9.7

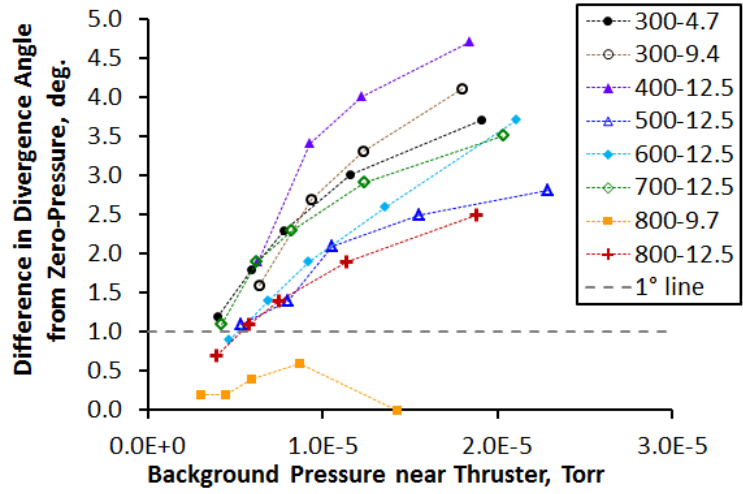

Figure 12. Difference in divergence angle from zero-pressure value as functions of background pressure.

throttle point, the data showed linear behavior up to about 1e-5 Torr, and then curved over to a gentler slope at higher background pressure. This figure indicates that data from testing done above 1e-5 Torr will likely give erroneous results when extrapolated to zero pressure. For this reason, the zero pressure projections shown in this paper were performed using data for background pressure up to 1e-5 Torr. If one now imposed the criteria that the calculated divergence angle must be within $1^{\circ}$ of the extrapolated zero-pressure value, as indicated by the grey dashed line, testing must be performed at below 4e-6 Torr. A background pressure of 4e-6 Torr was not achieved for some throttle points, implying that a $<1^{\circ}$ error criterion could not have been met by testing at the lowest achievable pressure alone. From the compiled data, the highest disagreement between the two methods for calculating divergence angle when testing below $1 \mathrm{e}-5$ Torr was $0.9^{\circ}$. The same for testing below $4 \mathrm{e}-6$ Torr was $0.2^{\circ}$. That is to say the FP analysis approach was self-consistent for both background pressure criteria.

For subsequent laboratory testing, if the $<1^{\circ}$ error is desired, the background pressure should be lower than $4 \mathrm{e}-6$ Torr. Alternately, testing must be performed at multiple background pressures that are lower than 1e-5 Torr and linearly extrapolated to zero pressure. For the purpose of SEP TDM flight hardware development, plume characterization should continue to be performed at multiple pressure even if $<4 \mathrm{e}-6$ Torr is achievable in order to obtain the best data possible for prediction of zero-pressure plume behavior. Note that the aforementioned guideline is based on plume characterization data only and different guideline will be shown for other criteria. The applicability of the different guidelines will be discussed in Section VI.

Across the throttle points, the general trend was that the extrapolated zero-pressure divergence angle was higher than measured divergence angle. Measurement obtained at high background pressure underestimated the true divergence angle of the HERMeS thruster and are not suitable for flight applications.

A full set of extrapolated zero-pressure current density profiles can be found in the appendix as Figs. 37 to 44 . One interesting observation about these figures was that the current density profiles at different distances almost overlap each other. The profile appeared to smooth out over distance but maintained largely the same amplitude and general shape. This observation implies that the effect of CEX interactions with background neutrals had been largely removed since the effect generally increased measured current density with distance. The observed smoothing was likely a result of CEX interactions with thruster neutrals and/or thruster plume evening out as it expands freely into vacuum.

For the purpose of performance characterization, the most important output of the RPA was the ion energy per charge of the main ion beam. This value is an indicator of the acceleration potential available to the ions for the purpose of thrust generation. Table 5 shows the ion energy per charge at different background pressures. To ensure only the main beam is examined, the data reported in this table was computed from the average of the results from polar angles of $-15^{\circ}, 0^{\circ}$, and $+15^{\circ}$. For reference, the ion energy per charge remains constant between polar angles of $-30^{\circ}$ and $+30^{\circ}$ to within $20 \mathrm{~V}$ for all tested throttle points. As stated in Section III.C, the reported values are based on $50 \%$ threshold-based averaging to strike a balance between excluding the CEX-broadened data and maintaining noise insensitivity. All data presented had been corrected for the local plasma potential. Values in the zero pressure row was linearly extrapolated using only data that were obtained at or below 1e-5 Torr background pressure. 
Table 5. Ion energy per charge at different background pressure.

\begin{tabular}{lcccccccc}
\hline \multicolumn{7}{c}{ lon energy per charge, V } \\
\hline & $\mathbf{3 0 0 - 4 . 7}$ & $\mathbf{3 0 0 - 9 . 4}$ & $\mathbf{4 0 0 - 1 2 . 5}$ & $\mathbf{5 0 0 - 1 2 . 5}$ & $\mathbf{6 0 0 - 1 2 . 5}$ & $\mathbf{7 0 0 - 1 2 . 5}$ & $\mathbf{8 0 0 - 9 . 7}$ & $\mathbf{8 0 0 - 1 2 . 5}$ \\
\hline P0X & 276.0 & 284.1 & 382.1 & 481.2 & 593.7 & 683.8 & 772.0 & 778.5 \\
P1X & 275.4 & 282.6 & 381.3 & 483.2 & 589.7 & 683.7 & 772.2 & 778.6 \\
P1.5X & 274.1 & 281.4 & 381.4 & 484.5 & 588.1 & 683.7 & 771.0 & 778.8 \\
P2X & 274.5 & 281.1 & 380.7 & 485.2 & 585.9 & 683.6 & 775.1 & 778.7 \\
P3X & 274.4 & 278.7 & 379.8 & 481.7 & 586.4 & 681.9 & 771.9 & 778.1 \\
\hline P5X & 273.0 & & & 481.6 & 585.5 & 681.8 & 769.9 & 776.3 \\
\hline
\end{tabular}

*The data in cell corresponding to 500-12.5 and P5X was from the condition 500-12.5-P4.5X. The data in cell corresponding to 600-12.5 and P5X was from the condition 600-12.5-P4.5X.

From Table 5, one can see that the ion energy per charge changed by very small amounts as the background pressure was varied. The maximum difference between measurements at the lowest and highest background pressures was about $5 \mathrm{~V}$, or on the order of the RPA accuracy. As such, background pressure did not have a significant influence on the ion energy per charge. No guidelines for maximum background pressure were derived based on ion energy per charge measurements.

Tables 6 and 7 show the current fractions from analysis of WFS data at different background pressure. All of the data shown in these tables were obtained at a polar angle of $0^{\circ}$ to ensure only the main beam was examined. Examination of Tables 6 and 7 indicates that the current fractions did not vary in any meaningful way as the background pressure was varied. The highest difference in the singly-charged current fraction between the lowest and highest tested background pressure was only 0.04 out of $\sim 0.7$. This level of difference was on the order of the uncertainty of the WFS. The largest change in the $\alpha_{\mathrm{m}}$ parameter from Eq. (7) was $\sim 0.015$, which was within the uncertainty of the WFS data analysis. Extrapolation of current fractions to zero pressure was not performed because data obtained at the lowest achievable background pressure was considered sufficient. No guidelines for maximum background pressure were derived based on the current fraction measurements.

Table 6. Current fractions at different background pressure for low discharge voltage conditions.

\begin{tabular}{lcccccccccccc}
\hline & \multicolumn{3}{c}{$\mathbf{3 0 0 - 4 . 7}$} & \multicolumn{4}{c}{$\mathbf{3 0 0 - 9 . 4}$} & \multicolumn{4}{c}{$\mathbf{4 0 0 - 1 2 . 5}$} & \multicolumn{3}{c}{$\mathbf{5 0 0 - 1 2 . 5}$} \\
\hline Current & & & & & & & & & & \\
Fraction: & $\mathrm{Xe}^{+}$ & $\mathrm{Xe}^{2+}$ & $\mathrm{Xe}^{3+}$ & $\mathrm{Xe}^{+}$ & $\mathrm{Xe}^{2+}$ & $\mathrm{Xe}^{3+}$ & $\mathrm{Xe}^{+}$ & $\mathrm{Xe}^{2+}$ & $\mathrm{Xe}^{3+}$ & $\mathrm{Xe}^{+}$ & $\mathrm{Xe}^{2+}$ & $\mathrm{Xe}^{3+}$ \\
\hline P1X & 0.67 & 0.28 & 0.06 & 0.56 & 0.37 & 0.07 & 0.52 & 0.38 & 0.10 & 0.51 & 0.33 & 0.17 \\
P1.5X & 0.66 & 0.29 & 0.04 & 0.56 & 0.38 & 0.06 & 0.53 & 0.37 & 0.10 & 0.51 & 0.33 & 0.16 \\
P2X & 0.66 & 0.28 & 0.05 & 0.53 & 0.39 & 0.08 & 0.49 & 0.39 & 0.12 & 0.51 & 0.33 & 0.16 \\
P3X & 0.66 & 0.29 & 0.05 & 0.54 & 0.38 & 0.08 & 0.52 & 0.39 & 0.09 & 0.51 & 0.32 & 0.16 \\
P5X $^{*}$ & 0.68 & 0.25 & 0.07 & & & & & & & 0.53 & 0.32 & 0.16 \\
\hline
\end{tabular}

*The data in cells corresponding to 500-12.5 and P5X was from the condition 500-12.5-P4.5X.

Table 7. Current fractions at different background pressure for high discharge voltage conditions.

\begin{tabular}{lccccccccccccc}
\hline \multicolumn{4}{c}{} & $\mathbf{6 0 0 - 1 2 . 5}$ & \multicolumn{4}{c}{$\mathbf{7 0 0 - 1 2 . 5}$} & \multicolumn{3}{c}{$\mathbf{8 0 0 - 9 . 7}$} & \multicolumn{4}{c}{$\mathbf{8 0 0 - 1 2 . 5}$} \\
\hline Current & & & & & & & & & & & \\
Fraction: & $\mathrm{Xe}^{+}$ & $\mathrm{Xe}^{2+}$ & $\mathrm{Xe}^{3+}$ & $\mathrm{Xe}^{+}$ & $\mathrm{Xe}^{2+}$ & $\mathrm{Xe}^{3+}$ & $\mathrm{Xe}^{+}$ & $\mathrm{Xe}^{2+}$ & $\mathrm{Xe}^{3+}$ & $\mathrm{Xe}^{+}$ & $\mathrm{Xe}^{2+}$ & $\mathrm{Xe}^{3+}$ \\
\hline P1X & 0.67 & 0.17 & 0.16 & 0.76 & 0.11 & 0.13 & 0.65 & 0.23 & 0.12 & 0.75 & 0.13 & 0.12 \\
P1.5X & 0.70 & 0.16 & 0.14 & 0.75 & 0.13 & 0.12 & 0.66 & 0.22 & 0.12 & 0.76 & 0.13 & 0.11 \\
P2X & 0.68 & 0.16 & 0.16 & 0.76 & 0.12 & 0.12 & 0.66 & 0.22 & 0.12 & 0.76 & 0.13 & 0.11 \\
P3X & 0.69 & 0.16 & 0.15 & 0.77 & 0.11 & 0.12 & 0.67 & 0.22 & 0.11 & 0.76 & 0.13 & 0.10 \\
P5X $^{\star}$ & 0.71 & 0.16 & 0.13 & 0.78 & 0.12 & 0.10 & 0.68 & 0.21 & 0.11 & 0.77 & 0.14 & 0.09 \\
\hline
\end{tabular}

*The data in cells corresponding to 600-12.5 and P5X was from the condition 600-12.5-P4.5X.

Thrust measurements were also linearly extrapolated to zero pressure and tabulated in Table 8. Examination of Table 8 shows that thrust was largely constant as functions of background pressure to within the measurement uncertainty except when background pressure was significantly higher than the lowest achievable pressure. At very high background pressure, thrust was noticeably lower than at other background pressure suggesting a change in thruster behavior. This data reinforces the notion that the thruster no longer behaved in a linear and predictable fashion when background pressure was too high. 
Figure 13 shows a plot of the percentage difference in thrust from the zero-pressure prediction as a function of background pressure. A grey dashed line denoting $1 \%$ is also shown in the figure for reference. With the exception of the 800-9.7 throttle point, thrust can be measured to within $1 \%$ of the extrapolated zero-pressure value if the background pressure is less than approximately 1e-5 Torr. Interestingly, the behavior of the thrust varied greatly between throttle points. For some throttle points such as 400-12.5 and 500-12.5, the thrust remained largely constant as background pressure was varied while for other throttle points the changes in thrust were more pronounced.

Since mass flow rate was also varied with background pressure to maintain a constant discharge power, examining only the thrust does not give a complete picture. Total efficiency was linearly extrapolated to zero pressure and tabulated in Table 9. Figure 14 shows the difference in total efficiency from the zero-pressure value as functions of background pressure. The total efficiency was calculated via Eq. (2) and linearly extrapolated to zero pressure the same way that thrust was. A grey dashed line denoting 0.01 is also shown in the figure for reference. With the exception of the 800-9.7 throttle point, the calculated total efficiency at pressures of up 1.2e-5 Torr had error of less than 0.01. Interestingly, throttle points such as 400-12.5 and 500-12.5 exhibited noticeable variation in total efficiency with background pressure while other throttle points exhibited negligible variations.

Table 8. Thrust at different background pressure.

\begin{tabular}{lcccccccc}
\hline \multicolumn{7}{c}{ Thrust, $\mathbf{~ m N}$} \\
\hline & $\mathbf{3 0 0 - 4 . 7}$ & $\mathbf{3 0 0 - 9 . 4}$ & $\mathbf{4 0 0 - 1 2 . 5}$ & $\mathbf{5 0 0 - 1 2 . 5}$ & $\mathbf{6 0 0 - 1 2 . 5}$ & $\mathbf{7 0 0 - 1 2 . 5}$ & $\mathbf{8 0 0 - 9 . 7}$ & $\mathbf{8 0 0 - 1 2 . 5}$ \\
\hline P0X & 312.2 & 572.5 & 682.4 & 627.3 & 595.2 & 543.4 & 345.5 & 523.9 \\
P1X & 310.5 & 572.0 & 682.5 & 627.9 & 592.6 & 542.0 & 339.3 & 521.8 \\
P1.5X & 309.9 & 570.0 & 683.0 & 629.3 & 591.6 & 541.7 & 336.7 & 522.3 \\
P2X & 308.9 & 569.5 & 682.7 & 628.7 & 590.2 & 540.8 & 336.9 & 520.2 \\
P3X & 308.0 & 567.6 & 681.7 & 629.0 & 588.5 & 539.9 & 328.4 & 518.7 \\
P5X & 303.5 & & & 626.7 & 583.1 & 535.6 & 316.0 & 509.0 \\
\hline
\end{tabular}

*The data in cell corresponding to 500-12.5 and P5X was from the condition 500-12.5-P4.5X. The data in cell corresponding to 600-12.5 and P5X was from the condition 600-12.5-P4.5X.

Table 9. Total efficiency at different background pressure.

\begin{tabular}{lcccccccc}
\hline \multicolumn{7}{c}{ Total Efficiency } \\
\hline & $\mathbf{3 0 0 - 4 . 7}$ & $\mathbf{3 0 0 - 9 . 4}$ & $\mathbf{4 0 0 - 1 2 . 5}$ & $\mathbf{5 0 0 - 1 2 . 5}$ & $\mathbf{6 0 0 - 1 2 . 5}$ & $\mathbf{7 0 0 - 1 2 . 5}$ & $\mathbf{8 0 0 - 9 . 7}$ & $\mathbf{8 0 0 - 1 2 . 5}$ \\
\hline P0X & 0.582 & 0.595 & 0.620 & 0.620 & 0.631 & 0.600 & 0.471 & 0.594 \\
P1X & 0.583 & 0.594 & 0.625 & 0.622 & 0.629 & 0.599 & 0.462 & 0.594 \\
P1.5X & 0.586 & 0.591 & 0.627 & 0.626 & 0.629 & 0.599 & 0.458 & 0.597 \\
P2X & 0.584 & 0.592 & 0.629 & 0.626 & 0.628 & 0.599 & 0.463 & 0.594 \\
P3X & 0.585 & 0.592 & 0.632 & 0.628 & 0.628 & 0.600 & 0.447 & 0.595 \\
\hline P5X & 0.578 & & & 0.628 & 0.623 & 0.597 & 0.428 & 0.583 \\
\hline
\end{tabular}

*The data in cell corresponding to 500-12.5 and P5X was from the condition 500-12.5-P4.5X. The data in cell corresponding to 600-12.5 and P5X was from the condition 600-12.5-P4.5X.

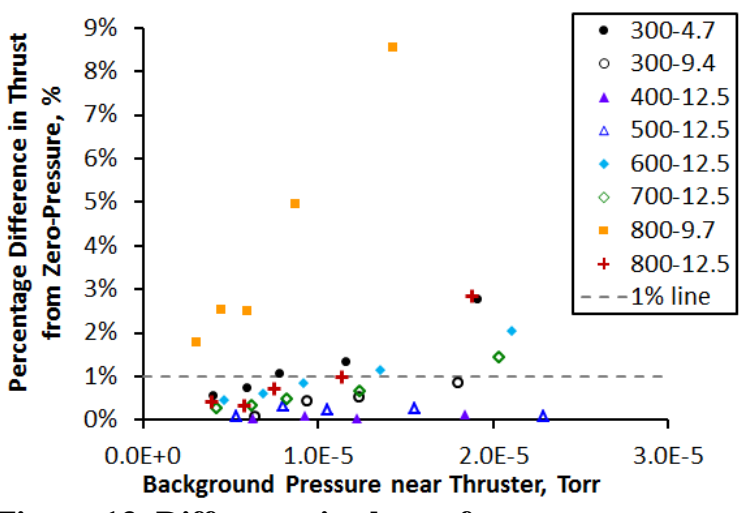

Figure 13. Difference in thrust from zero-pressure value as functions of background pressure.

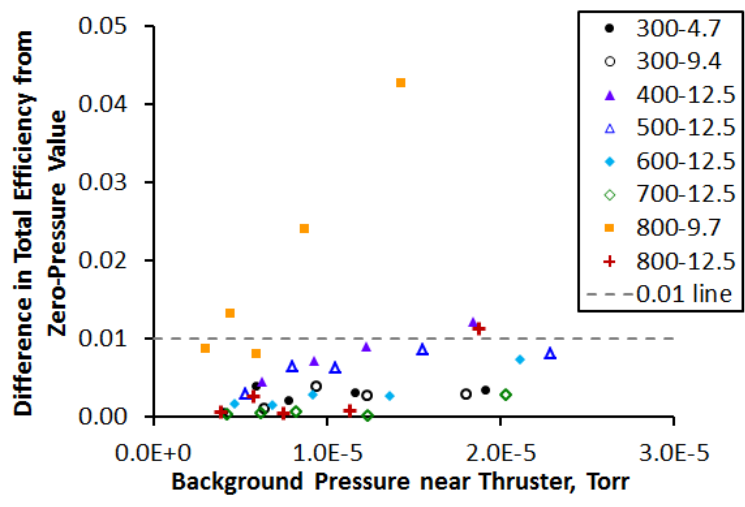

Figure 14. Difference in total efficiency from zeropressure value as functions of background pressure.

14

American Institute of Aeronautics and Astronautics 
After examining the performance characterization results from all of the plasma diagnostics, the best course of action for future HERMeS testing of similar type is to test at multiple background pressure, below $\sim 1 \mathrm{e}-5$ Torr. Data taken at background pressure above 1e-5 Torr trend in a nonlinear fashion with pressure and can make any projection erroneous.

Table 10 summarizes the analysis results of the probe package when linearly extrapolated to zero pressure. HERMeS TDU1 appears to perform most efficiently at the 600-12.5 throttle point and tends to behave poorly at high-voltage, low-current throttle point such as $800-9.7$. We speculate that the current density was too low at 800 9.7 to maintain a stable discharge.

Table 10. Summary of probe package analysis results linearly extrapolated to zero pressure.

\begin{tabular}{lccccccc}
\hline & Thrust & $\boldsymbol{\eta}_{\text {tot }}$ & $\boldsymbol{\eta}_{\mathbf{d}}$ & $\boldsymbol{\eta}_{\mathbf{b}}$ & $\boldsymbol{\eta}_{\mathbf{v}}$ & $\boldsymbol{\eta}_{\mathbf{q}}$ & $\boldsymbol{\eta}_{\mathbf{m}}$ \\
\hline $\mathbf{3 0 0 - 4 . 7}$ & 312 & 0.582 & 0.836 & 0.865 & 0.920 & 0.972 & 0.926 \\
$\mathbf{3 0 0 - 9 . 4}$ & 573 & 0.595 & 0.800 & 0.855 & 0.947 & 0.967 & 1.023 \\
$\mathbf{4 0 0 - 1 2 . 5}$ & 682 & 0.620 & 0.817 & 0.860 & 0.955 & 0.964 & 0.989 \\
$\mathbf{5 0 0 - 1 2 . 5}$ & 627 & 0.620 & 0.876 & 0.833 & 0.962 & 0.958 & 0.860 \\
$\mathbf{6 0 0 - 1 2 . 5}$ & 595 & 0.631 & 0.882 & 0.883 & 0.990 & 0.964 & 0.979 \\
$\mathbf{7 0 0 - 1 2 . 5}$ & 543 & 0.600 & 0.875 & 0.863 & 0.977 & 0.971 & 0.956 \\
$\mathbf{8 0 0 - 9 . 7}$ & 346 & 0.471 & 0.820 & 0.823 & 0.965 & 0.966 & 0.899 \\
$\mathbf{8 0 0 - 1 2 . 5}$ & 524 & 0.594 & 0.878 & 0.876 & 0.973 & 0.971 & 0.924 \\
\hline
\end{tabular}

In general, the HERMeS TDU1 produced less thrust and required less propellant to maintain constant discharge power as the background pressure increased. For the most part, the decrease in thrust and mass flow rates were such that the total efficiency did not change significantly. The small changes in total efficiency that was observed did not trend in a consistent direction with background pressure. As the background pressure was increased, the divergence angle of the thruster plume decreased. At the same time, the ion energy per charge slightly decreased but the decrease was on the order of the measurement uncertainty. Any change in charge species composition with background pressure was also negligibly small. Close examination of the FP data and performance trend revealed that the thruster and plasma plume began showing signs of non-linear behavior at background pressure in excess of 1e-5 Torr. Any linear neutral ingestion model should only be applied on data obtained in low background pressure $(<1 \mathrm{e}-5$ Torr $)$.

\section{B. Oscillation Results}

Figures 15 to 17 show several examples of phi-t diagrams from thruster HSC analysis. A phi-t diagram is the equivalent of an $\mathrm{x}$-t diagram for visualizing traveling waves in a cylindrical or spherical coordinate system. The horizontal axis is the azimuthal angle and the vertical axis is time. Red pixels represent high light intensity and blue pixels represent low light intensity. These figures illustrate that the HERMeS TDU1 discharge channel exhibited only global oscillation. On a phi-t diagram, spokes mode appear as diagonal bands because the angular position of

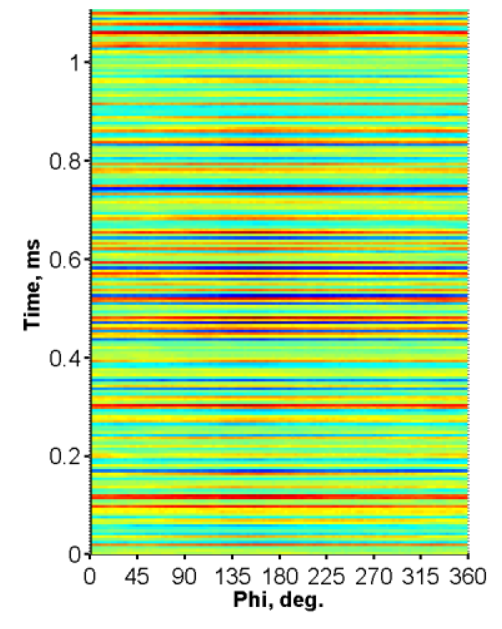

Figure 15. Thruster phi-t diagram for 300-9.4-P1X.

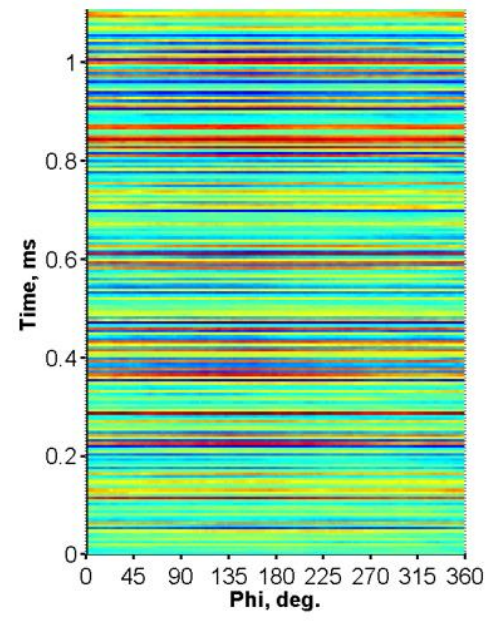

Figure 16. Thruster phi-t diagram for 300-9.4-P3X.

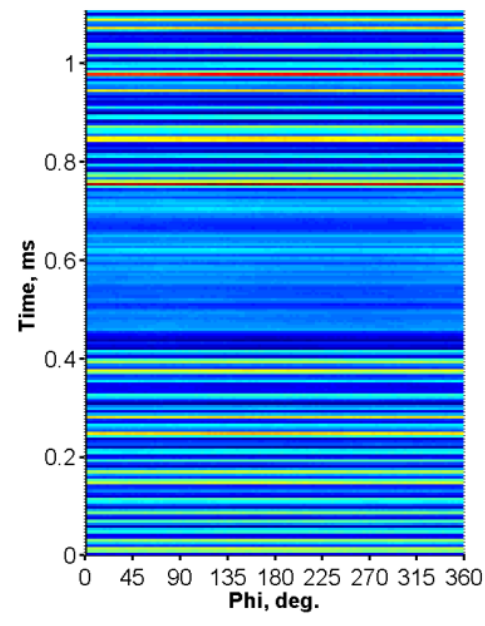

Figure 17. Thruster phi-t diagram for 800-12.5-P1X. 
spokes changes with time. The appearance of horizontal bands indicated that the entire discharge channel oscillated together.

In contrast, Figs. 18 to 20 show several examples of phi-t diagrams from cathode HSC analysis. Diagonal bands are clearly visible in these phi-t diagrams indicating the presence of a strong $\mathrm{m}=1$ mode that corresponds to the cathode gradient-driven mode.

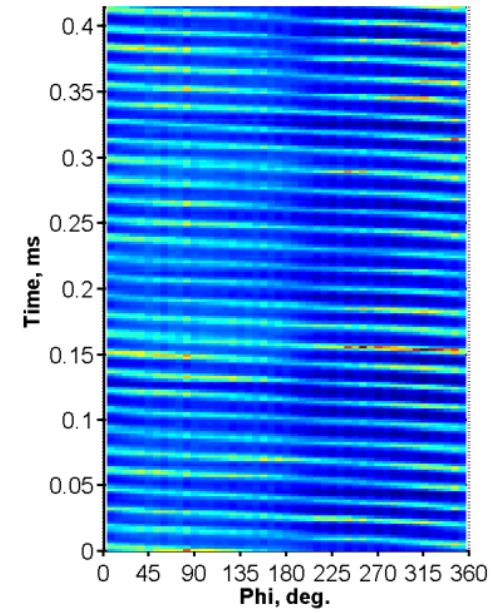

Figure 18. Cathode phi-t diagram for 300-9.4-P1X.

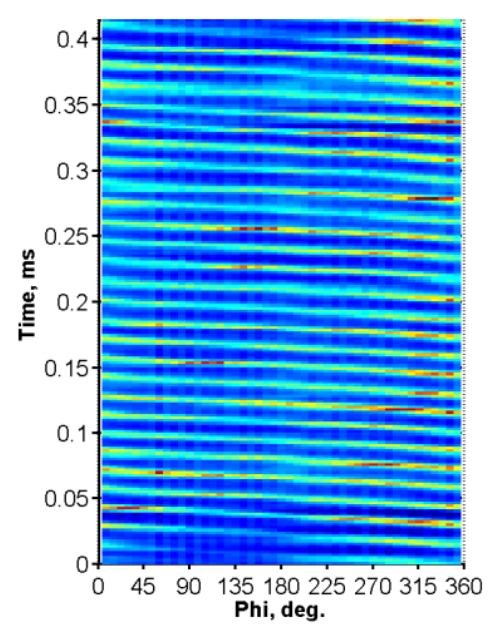

Figure 19. Cathode phi-t diagram for 300-9.4-P3X.

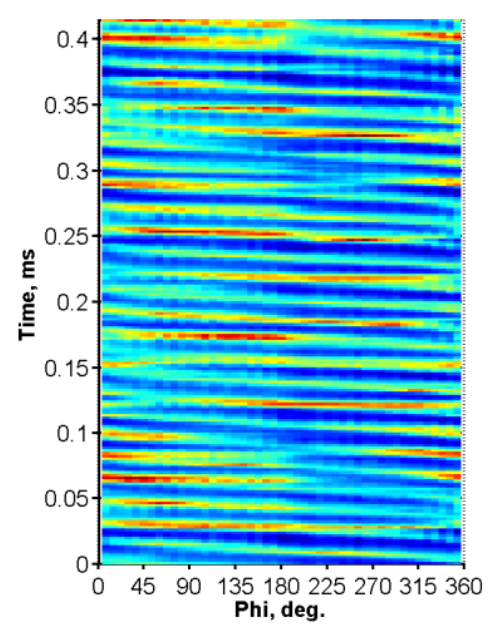

Figure 20. Cathode phi-t diagram for 800-12.5-P1X.

To better visualize trends in the oscillation modes with background pressure, a series of probability density functions (PDFs) of the light intensity were generated in addition to the power spectra. A PDF of light intensity is a good way to visualize how often the plasma is bright as opposed to dark as well as how bright and how dark the plasma gets. Different types of plasma oscillations have different PDF characteristics. For example, a truly random oscillation process exhibits a Gaussian PDF distribution while a purely sinusoidal oscillation process exhibits a unique two-peak PDF distribution. For the data during the FECT, cathode PDFs displayed the same general shape as the corresponding thruster PDFs.

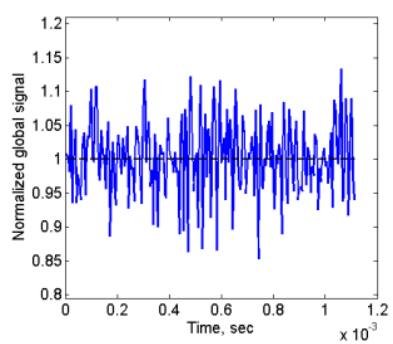

Figure 21. Thruster intensity (left) and probability distribution function (right) for 300-9.4-P1X.
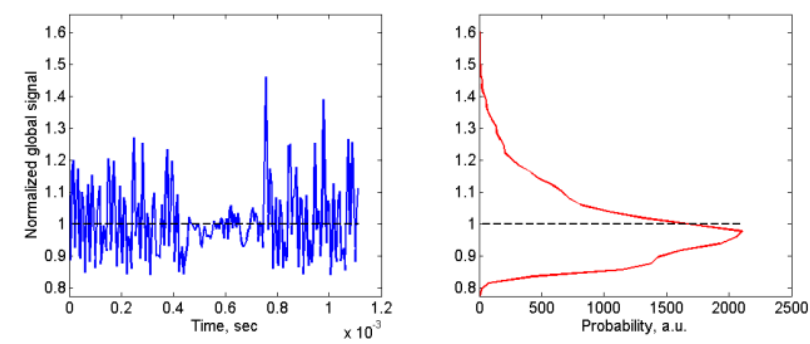

Figure 23. Thruster intensity (left) and probability distribution function (right) for 800-12.5-P1X.
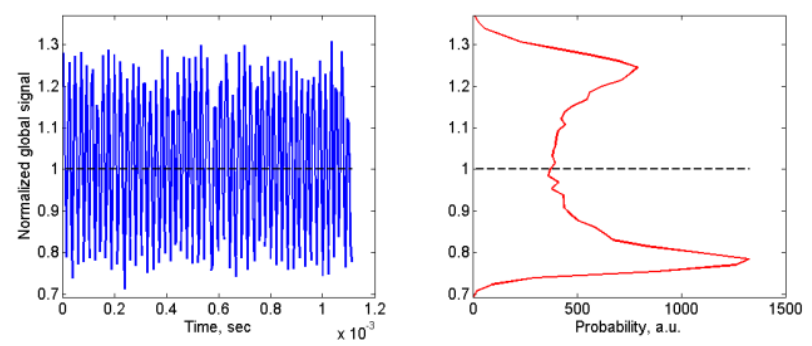

Figure 22. Thruster intensity (left) and probability distribution function (right) for 600-12.5-P1X.
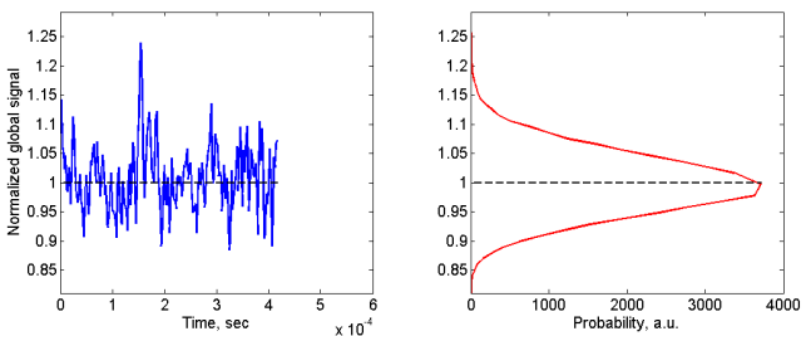

Figure 24. Cathode intensity (left) and probability distribution function (right) for 300-9.4-P1X. 
Figures 21 to 23 show examples of different patterns of global oscillations observed during the FECT. The left sub-plot of each figure shows a portion of the light intensity as a function of time. The right sub-plot shows the associated PDF. Figure 21 shows an example of a PDF with Gaussian shape where the light intensity appears to oscillate randomly. Figure 22 shows an example of a PDF with a unique two-peak shape that is only seen when the oscillation is purely sinusoidal. Figure 23 shows an example of a PDF with a skewed shape. A skewed PDF was found to be associated with a discharge that periodically flares to high intensities for very short durations and is at a low intensity most of the time. Figure 24 shows an example of the pattern observed in the plasma near the cathode during testing. The combination of Figs. 21 and 24 illustrate the similarity in the PDFs between the thruster and cathode videos for a given operating condition.

Figures 25 and 26 show examples of thruster light intensity power spectra observed during the FECT. The $\mathrm{M}=0$ spectra corresponds to global oscillations and dominates the spectra for all tested throttle points. Figures 27 and 28 show examples of cathode light intensity power spectra observed during the FECT. Generally speaking, the $\mathrm{M}=0$ cathode spectra match the corresponding thruster spectra while the $M=1$ cathode spectra display a unique gradientdriven mode.

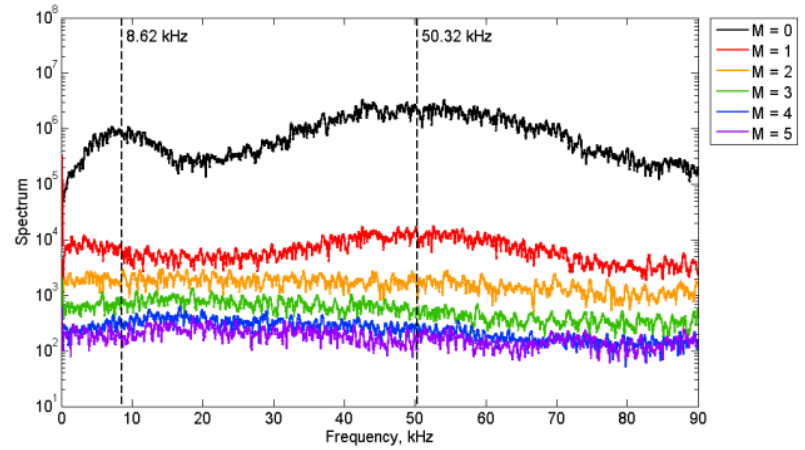

Figure 25. Thruster light intensity power spectra for 300-9.4-P1X.

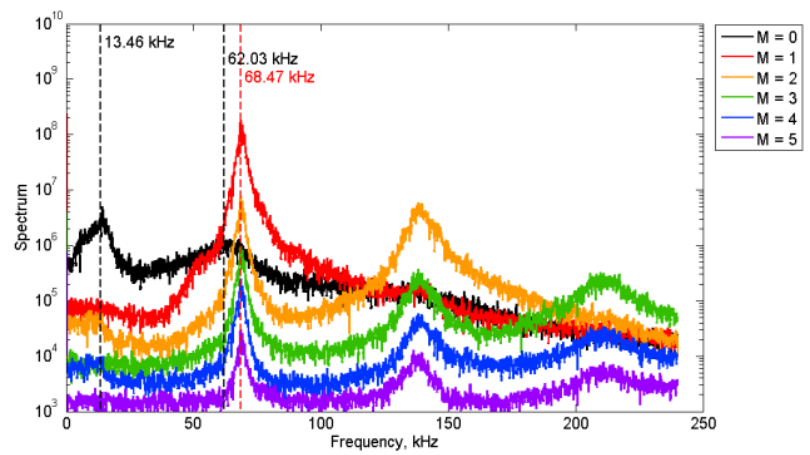

Figure 27. Cathode light intensity power spectra for 300-9.4-P1X.

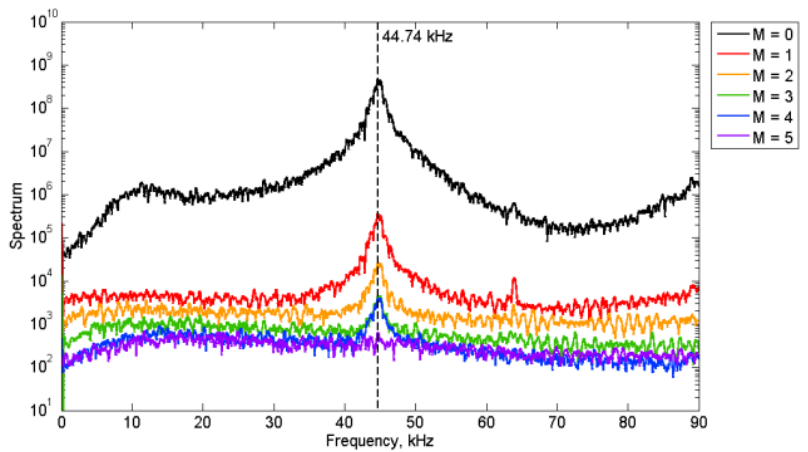

Figure 26. Thruster light intensity power spectra for 600-12.5-P1X.

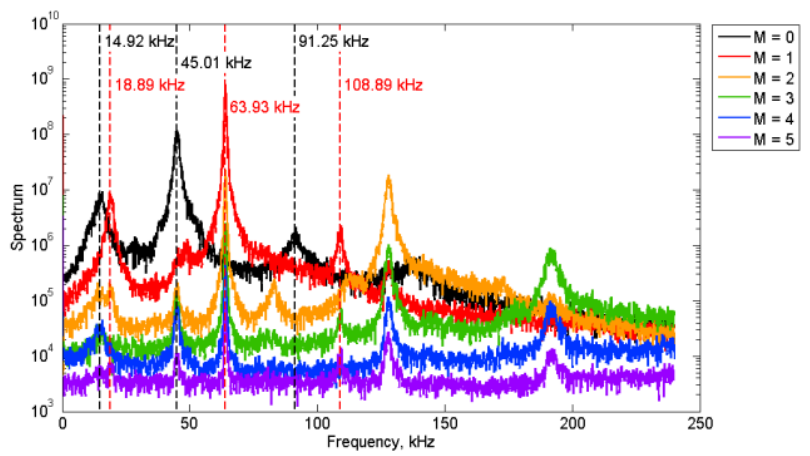

Figure 28. Cathode light intensity power spectra for 600-12.5-P1X

From Figures 25 to 26, one can see that there were both low frequency $(\sim 10 \mathrm{kHz})$ and high frequency (40-60 $\mathrm{kHz}$ ) global oscillations occurring in the discharge channel. For a few of the conditions, there were also low amplitude harmonics of the main peaks present.

To gain a better understanding of the nature of the global oscillation modes present, a cross-correlation study involving the PDF and power spectra data was performed. Operating conditions were categorized under one of three types according to their PDFs: Gaussian, Skewed, or Sinusoid. Operating conditions were also categorized under one of three categories according to their power spectra: low frequency peak only, high frequency peak only, low and high frequency peaks present. In order to increase the sample size, the cross-correlation study was performed with additional

Table 11. Result of cross correlation study involving thruster PDF and power spectra data.

\begin{tabular}{lccc}
\hline & Gaussian & Skewed & Sinusoid \\
\hline $\begin{array}{l}\text { Low freq. } \\
\text { only }\end{array}$ & $10 \%$ & $21 \%$ & $2 \%$ \\
$\begin{array}{l}\text { High freq. } \\
\text { only }\end{array}$ & $13 \%$ & $3 \%$ & $21 \%$ \\
$\begin{array}{l}\text { Low and } \\
\text { high freq. }\end{array}$ & $10 \%$ & $15 \%$ & $5 \%$ \\
\hline
\end{tabular}


HSC data from the performance testing that took place with the same set up as the FECT. More information regarding the HSC data from the performance testing is in a separate publication. ${ }^{38}$ Table 11 summarizes the results of the correlation study. The percentage in each cell is the percent of total number of operating conditions that fall under the associated category. For example, $21 \%$ of the operating conditions studied with the HSC had sinusoidal PDFs and high frequency global oscillation. The sample size of the study was 68 . From this table, one can see that sinusoidal PDFs were predominately associated with high frequency global oscillation modes while skewed PDFs were predominately associated with low frequency global oscillation modes. Gaussian PDFs were associated with both low and high frequency oscillation modes. The frequency, PDF, and location of the low frequency Gaussian mode suggests that it is the breathing mode commonly seen in Hall thrusters with both magnetically shielded and unshielded topologies. ${ }^{37,}$, 39-42 The high frequency global oscillation with a sinusoidal PDF does not appear to match any oscillation mode previously described in the literature and may be unique to magnetically-shielded Hall thrusters. Cathode gradient-driven mode was also found in the cathode HSC data. Since a detailed study of oscillation physics was not the purpose of the FECT, more detail and speculations about these oscillation modes are left to a separate publications dedicated to analysis of HSC data. ${ }^{38}$

To determine how the oscillation characteristics changed with background pressure a number of contour plots were generated. Figures 29 and 30 show two representative contour plots of the PDFs of the thruster light intensity as the background pressure varied. In PDF contours, red represents high probability, blue represents low probability, and a pair of dashed lines indicate the approximate boundaries the light intensity. The dashed lines were calculated by determining where the PDF dropped below 1\% of maximum probability. Figure 29 shows the 300-9.4 throttle point and represents the basic trend exhibited at most throttle point, which was that the PDF did not change meaningfully as the background pressure varied. Figure 30 shows the $800-12.5$ throttle point and is unique in that the PDF became more skewed as the background pressure was increased.

Figures 31 and 32 show two representative contour plots of the $m=0$ power spectra of the thruster light intensity

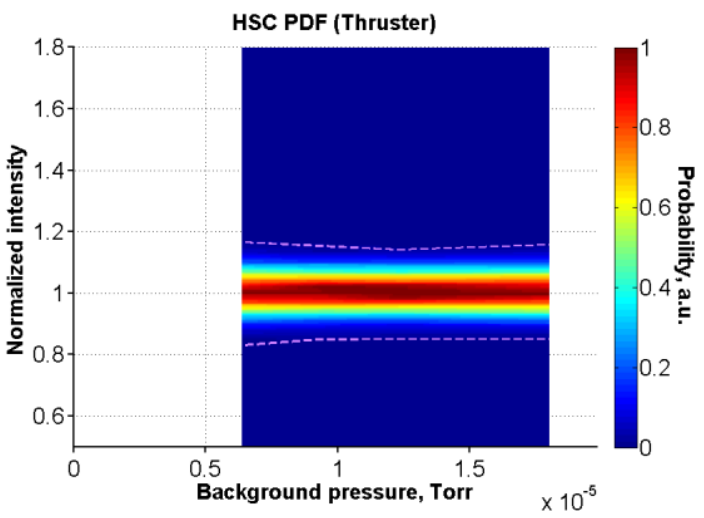

Figure 29. Contour plot of the PDF of the thruster light intensity as background pressure varied for the 300-9.4 throttle point.

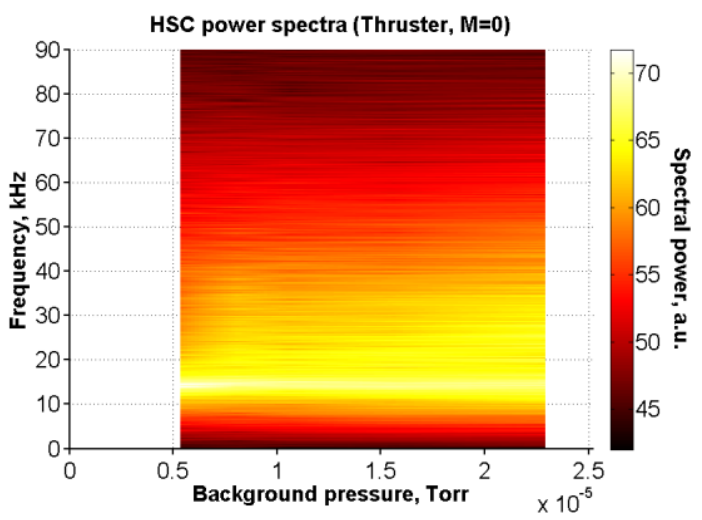

Figure 31. Contour plot of the $m=0$ power spectra of the thruster light intensity as background pressure varied for the 500-12.5 throttle point.

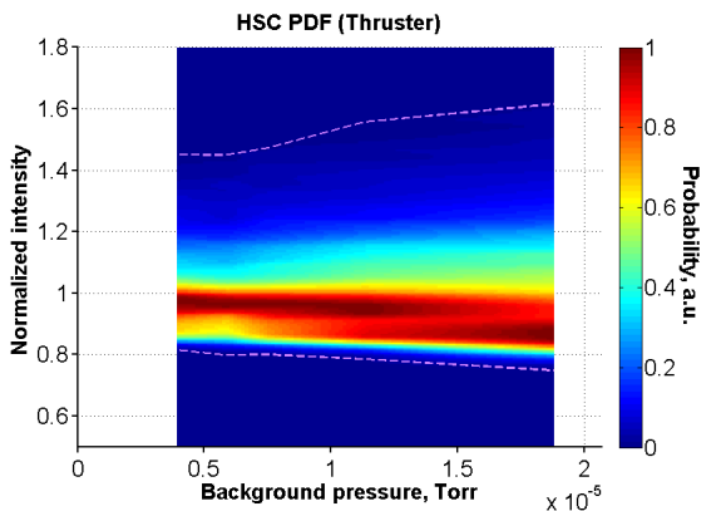

Figure 30. Contour plot of the PDF of the thruster light intensity as background pressure varied for the 800-12.5 throttle point.

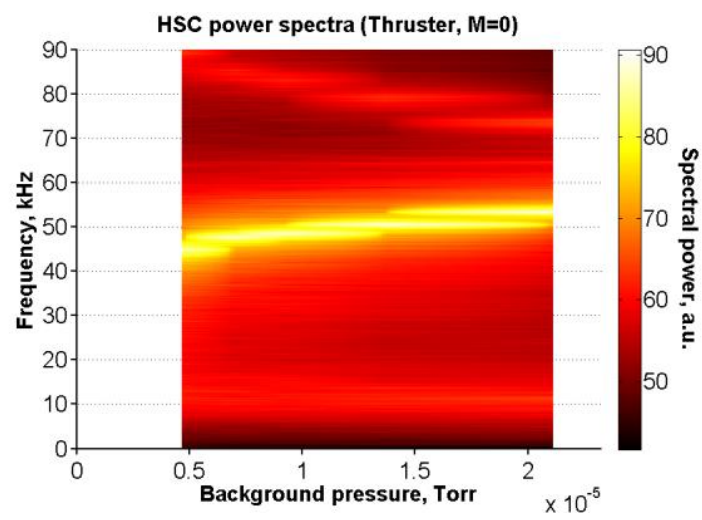

Figure 32. Contour plot of the $m=0$ power spectra of the thruster light intensity as background pressure varied for the $600-12.5$ throttle point.

18

American Institute of Aeronautics and Astronautics 
as the background pressure varied. In power spectra contours, yellowish white represents peaks and dark red represents trough. The color bar is on a base-10 decibel scale so a difference of 10 on the color bar is a factor of 10 in the absolute magnitude of the spectral power. Due to the limited number of pressures at which data was taken, the peaks will occasionally take on a banded appearance. These discrete bands are purely artifacts of the limited resolution in background pressure. The actual peaks are generally continuous functions of background pressure. Figure 31 shows the 500-12.5 throttle point and represents the trend exhibited at most throttle point, which was that the power spectra did not change meaningfully over the tested range of background pressure. Figure 32 shows the 600-12.5 throttle point and represents the trend exhibited at three throttle points where the high frequency peak increased in frequency with background pressure.

Table 12 summarizes the general trend of the thruster PDF and power spectra as functions of background pressure. This table shows that there were some differences in behavior at different throttle points. However, none of the PDF shape changed to a different type with varying background pressure and the same spectral peaks present at low background pressure were still present at high background pressure, implying that the oscillation mode of thruster did not change over the range of background pressure tested.

Table 12. General trend of the thruster PDF and power spectra with increasing background pressure.

\begin{tabular}{ccc}
\hline Throttle point & Trend in PDF & Trend power spectra \\
\hline $\mathbf{3 0 0 - 4 . 7}$ & Constant & Constant \\
$\mathbf{3 0 0 - 9 . 4}$ & Constant & Increase in high frequency peak \\
$\mathbf{4 0 0 - 1 2 . 5}$ & Constant & Increase in high frequency peak \\
$\mathbf{5 0 0 - 1 2 . 5}$ & Constant & Constant \\
$\mathbf{6 0 0 - 1 2 . 5}$ & Constant & Increase in high frequency peak \\
$\mathbf{7 0 0 - 1 2 . 5}$ & Nearly constant & Constant \\
$\mathbf{8 0 0 - 9 . 7}$ & Constant & Constant \\
$\mathbf{8 0 0 - 1 2 . 5}$ & Increasingly skewed & Slight decrease in high frequency peak \\
\hline
\end{tabular}

For the purpose of the FECT, the small change in oscillation frequency found in the HSC data was not a cause for concern because the amplitude of the oscillations, as shown in the PDFs, were mostly constant. The lone exception of 800-12.5 bears closer scrutiny. An examination of Figure 30 shows that the changes in the PDF became more prominent starting at just below 1e-5 Torr. Since the spectral peaks for 800-12.5 were mostly constant, we conclude that testing at $<1 \mathrm{e}-5$ Torr is sufficient for capturing the oscillation characteristics of the HERMeS TDU1.

Examination of the cathode $m=1$ power spectra showed that the frequency of the gradient-driven instability was constant with varying background pressure. The cathode PDF and $\mathrm{m}=0$ power spectra were in excellent agreement with the thruster PDF and $\mathrm{m}=0$ power spectra and are not shown.

Additional contour plots from the HSC data analysis can be found in the Appendix. A full set of contour plots of the PDFs of the thruster light intensity versus the background pressure is shown in Figs. 45 to 52 . A full set of contour plots of the $\mathrm{m}=0$ power spectra of the thruster light intensity versus the background pressure is shown in Figs. 53 to 60 . A full set of contour plots of the $\mathrm{m}=1$ power spectra of the cathode light intensity versus the background pressure is shown in Figs. 61 to 68.

\section{Plume Results for Spacecraft Interaction Studies}

Addressing the second primary objective (obtain plasma data for spacecraft interaction) of the test require close examination of different aspects of the plasma plume data. Specifically, information regarding the current density and energy distribution of the ions in the wing of the plume is needed to establish stay-out zones for spacecraft components.

Figure 33 shows the current density profiles at the wing of the plasma plume for the 800-12.5 throttle point at varying background pressure. Results shown in this figure were taken approximately one meter away from the thruster. Figure 33 illustrates the difficulty associated with obtaining plume data for spacecraft interaction studies. Even at the lowest background pressure achieved (3.9e-6 Torr for this throttle point), there was still a large difference between the

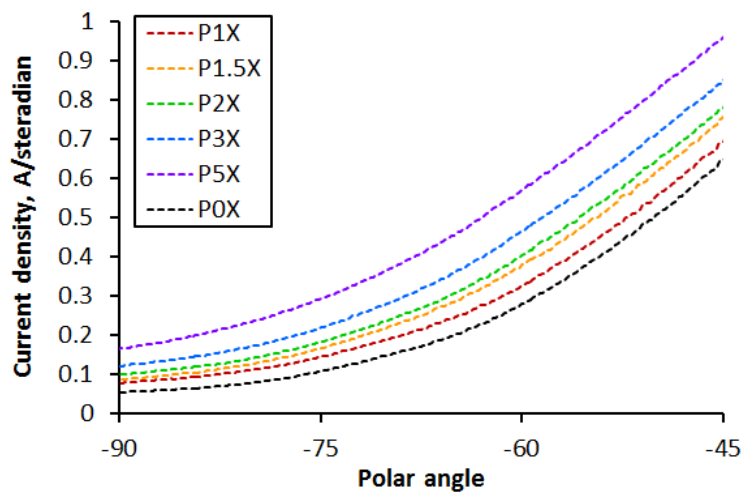

Figure 33. Current density profiles at the wing of the plasma plume for the 800-12.5 throttle point at varying background pressure. 
measured current density and the extrapolated zero-pressure value. For example, at a polar angle of $-90^{\circ}$, the current density for the P1X operating condition was $45 \%$ higher than the extrapolated zero-pressure value. At a polar angle of $-60^{\circ}$, the P1X value was $17 \%$ higher than the extrapolated zero-pressure value. The general trend across all throttle points was that the further away one moves from the firing axis (i.e. the larger the magnitude of the polar angle) the greater the variation in current density with background pressure. One explanation for this trend is that the current density associated with CEX has a flatter, much more divergent angular distribution than the main beam. While current density associated with CEX scales largely with the background pressure the current density associated with the main beam does not. At the wing, contribution to current density is dominated by CEX, while at the center, current density is dominated by the main beam. Thus at polar angles far from $0^{\circ}$, the current density is highly influenced by background neutrals. Based on examination of the data taken during the FECT, in order to obtain current density measurements at the $\pm 90^{\circ}$ location to within $10 \%$ of the extrapolated zero-pressure value, one would have to operate with a background pressure of less than 1e-6 Torr. Such a low background is not currently accessible and may not be practically achievable. Instead, a prediction of the zero-pressure profile can be obtained by either measuring the current density at multiple background pressure and extrapolating to zero pressure or by employing a physics-based plume model calibrated with empirical data. The team has settled on latter approach for spacecraft interaction studies. The plume model will be described in Section V.

In addition to current density, another important piece of information for improving the plume model is the energy distribution. In particular, focus was placed on the presence of high-angle high-energy ions after their discovery during testing of other Hall thrusters. ${ }^{15,}{ }^{43}, 44$ RPA data were taken at high polar angles to provide that information. Figure 34 shows the ion energy per charge profiles for the 300-9.4 throttle point at a polar angle of $-90^{\circ}$ and at varying background pressure. Figure 35 shows the same for the $800-12.5$ throttle point at $-60^{\circ}$. For reference, no high-energy ions were detected at $\pm 90^{\circ}$ for the 800-12.5 throttle point. From these two figures, one can see that the presence of high-energy ions was readily detected even at elevated background pressure. However, the amplitude of the high-energy ion peak varied greatly with background pressure. Examination of the data set revealed that at the lowest background pressure achieved, the height of the high-energy peak measured in the wing of the plume can be up to $20 \%$ too low. Thus, to quantify the amount of high-energy ion versus low-energy ion for the purpose of spacecraft interaction study, obtaining data at multiple background pressures is necessary. Furthermore, ion energy per charge as a function of polar angle was very helpful in calibrating the plume model because the divergence of the main beam is closely related to the location of the acceleration zone in the discharge channel of a Hall thruster.
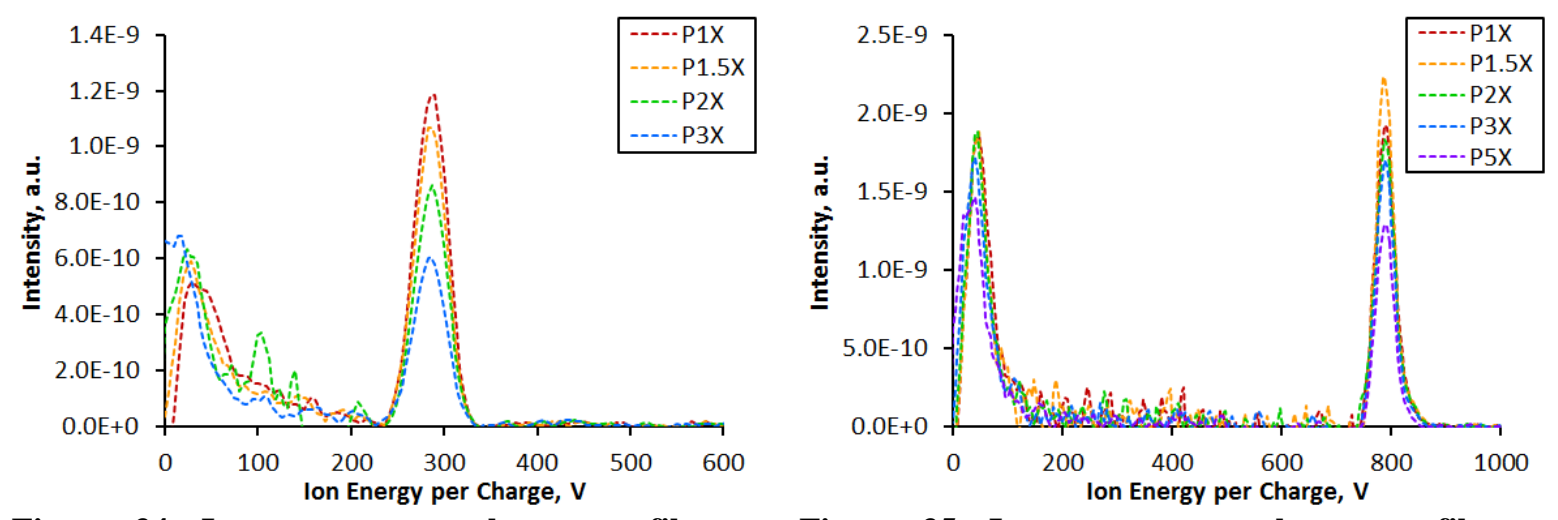

Figure 34. Ion energy per charge profiles at various background pressures for the 300-9.4 throttle point at a polar angle of $\mathbf{- 9 0 ^ { \circ }}$.

Figure 35. Ion energy per charge profiles at various background pressures for the 800-12.5 throttle point at a polar angle of $-\mathbf{6 0}^{\circ}$.

Additional plots of ion energy per charge at different background pressure can be found in the Appendix as Figs. 69 to 71 . A comparison of the plume data with the plume model will be shown in Section V.

\section{Plume Model, Results, and Comparisons}

This section begins with a description of the plume model. HallPlume2D expands the solution of Hall2De, a first-principles code that models the plasma discharge in the interior and in the near-field of a Hall thruster, to distances in excess of 30 meters from the thruster. ${ }^{45}$ The computational domain of HallPlume2D overlaps that of Hall2De in the near-field. This overlap allows for a smooth transition between the plasma properties in Hall2De and 
HallPlume2D. Computational cells are generated by considering a set of circles and rays that are orthogonal with each other.

HallPlume2D takes advantage of the methods already implemented in Hall2De but solves a set of simplified equations, which allow for faster computation times. Equations of motion are solved independently for ions, electrons, and neutrals. Ions are treated using a hydrodynamics approach. Fluid approximation is used to circumvent the difficulty of modeling the motion of ions with very distinct energies. Multiple ion populations that group ions of similar energy, referred to here as "fluids", can be defined in the algorithm. A typical example of this approach is to define two fluids in the simulation with threshold energy per charge of $50 \mathrm{~V}$ dividing the two for a thruster operating at a discharge voltage of $300 \mathrm{~V}$. Under these conditions, Hall2De treats the ions generated in the acceleration channel as belonging to "fluid 1" while the ions generated in regions where the plasma potential is below $50 \mathrm{~V}$ as belonging in "fluid 2". These two populations will follow their own equations of motion and only interact with one another through charge exchange collisions (i.e., in the previous example, if a fast ion undergoes a CEX collision with a neutral in a region where the plasma potential is below $50 \mathrm{~V}$, the population of fast ions loses one ion that is transferred to the population of slow ions). Finally, each "fluid" can contain singly, doubly, and triply charged ions. HallPlume2D uses the density and momentum of each of the ion fluid populations in the near-field of the thruster and expands the solution into the far-field. For each species, density and momentum are computed using the isothermal hydrodynamics equations (Eq. (11) to (14)) in the presence of an electric field.

$$
\begin{aligned}
& \frac{\partial \mathrm{n}_{\mathrm{iC}, \mathrm{iF}}}{\partial \mathrm{t}}+\nabla \cdot\left(\mathrm{n}_{\mathrm{iC}, \mathrm{iF}} \mathbf{u}_{\mathrm{iC}, \mathrm{iF}}\right)=\dot{\mathrm{n}}_{\mathrm{iC}, \mathrm{iF}}, \\
& \dot{\mathrm{n}}_{\mathrm{iC}, \mathrm{iF}}=\mathrm{b}_{\mathrm{iF}}(\phi)\left(\dot{\mathrm{n}}_{\mathrm{iz}, 0 \rightarrow \mathrm{i}, \mathrm{iF}}+\sum_{\mathrm{jF}=1, \mathrm{nF}} \dot{\mathrm{n}}_{\mathrm{CEX}, \mathrm{iC}, \mathrm{jF}}\right)-\dot{\mathrm{n}}_{\mathrm{CEX}, \mathrm{iC}, \mathrm{iF}}+\sum_{\mathrm{jC}<\mathrm{iC}} \dot{\mathrm{n}}_{\mathrm{iz}, \mathrm{jC} \rightarrow \mathrm{iC}, \mathrm{iF}}-\sum_{\mathrm{jC}>\mathrm{iC}} \dot{\mathrm{n}}_{\mathrm{iz}, \mathrm{iC} \rightarrow \mathrm{jC}, \mathrm{F} F}, \\
& \frac{\partial}{\partial \mathrm{t}}(\mathrm{nu})_{\mathrm{i} C, \mathrm{FF}}+\nabla \cdot(\mathrm{nuu})_{\mathrm{iC}, \mathrm{iF}}=\frac{\mathrm{q}_{\mathrm{iC}} \mathrm{n}_{\mathrm{iC}, \mathrm{iF}} \mathbf{E}}{\mathrm{m}}-\frac{\mathrm{kT}_{\mathrm{i}}}{\mathrm{m}_{\mathrm{i}}} \nabla\left(\mathrm{n}_{\mathrm{iC}, \mathrm{F}}\right)+\mathbf{R}_{\text {inelastic,iC,iF}},
\end{aligned}
$$

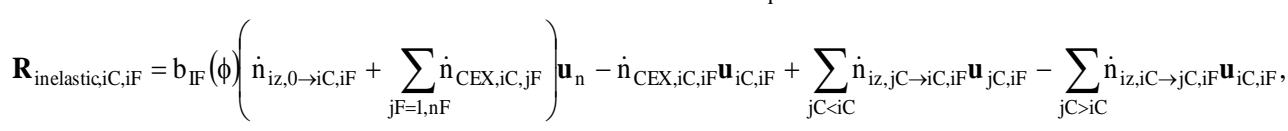

Where $\mathrm{iC}, \mathrm{iF}$ denote the charge state (i.e., singly-, doubly-, triply-charged ions, 1, 2, and 3, respectively) and the "fluid" number (up to 4), respectively. $\mathrm{n}$ is the number density, $\mathbf{u}$ is the velocity field, $\mathrm{m}$ is the ion atomic mass, $\mathrm{k}$ is Boltzmann's constant, $\mathrm{q}_{\mathrm{iC}}$ is the charge of an ion particle in Coulombs, and $\mathrm{T}_{\mathrm{i}}$ is the ion temperature, considered isothermal and equal to the temperature of the thruster walls. The ion production term, $\dot{\mathrm{n}}$, and the inelastic drag, $\mathbf{R}_{\text {inelastic }}$, contain the implementation of the multi-fluid algorithm through the $b_{\mathrm{iF}}(\phi)$ function. $b_{\mathrm{iF}}(\phi)=1$ if the plasma potential at the location in the computational domain where the equation is evaluated falls within the bracket defined for $\mathrm{iF}$, and $\mathrm{b}_{\mathrm{iF}}(\phi)=0$ otherwise. In the far-field, $\mathrm{b}_{\mathrm{iF}}$ is typically 0 for fluid 1 (i.e., the fast ions) and 1 for fluid 2 (i.e., the slow ions). If $b_{\mathrm{iF}}=1$, the population $\mathrm{iC}, \mathrm{iF}$ increases its density thanks to electron-impact ionization of neutrals, $\dot{\mathrm{n}}_{\mathrm{iz}, 0 \rightarrow \mathrm{iC}, \mathrm{iF}}$. Charge exchange reactions undergone by ions of any population with the same charge $\mathrm{iC}$ also result in an increase, given by the sum of the charge exchange rates $\dot{\mathrm{n}}_{\mathrm{CEX}, \mathrm{iC}, \mathrm{F} \text {, }}$, in the density of the population $\mathrm{iC}, \mathrm{iF}$ when $\mathrm{b}_{\mathrm{iF}}(\phi)=1$. Each population $\mathrm{iC}, \mathrm{iF}$ also losses ions through charge exchange (which are recovered if $\left.\mathrm{b}_{\mathrm{iF}}(\phi)=1\right)$ and through electron-impact ionization to charge states higher than $\mathrm{iC}$, and gains ions through the latter mechanism from charge states lower than $\mathrm{iC}\left(\dot{\mathrm{n}}_{\mathrm{iz}, \mathrm{j} C \rightarrow \mathrm{iC}, \mathrm{iF}}\right.$ terms). The change in fluid momentum due to these reactions is consistently reflected in the inelastic drag term $\mathbf{R}_{\text {inelastic,ic,iF, }}$, where $\mathbf{u}_{\mathrm{n}}$ is the velocity of the neutrals. Ionization rates are computed using the expression shown in Eq. (15).

$$
\dot{\mathrm{n}}_{\mathrm{iz}, \mathrm{jC} \rightarrow \mathrm{iC}, \mathrm{iF}}=\mathrm{n}_{\mathrm{e}} \mathrm{n}_{\mathrm{jC}, \mathrm{iF}} \overline{\mathrm{c}}_{\mathrm{e}} \sigma_{\mathrm{jC}, \mathrm{iC}}
$$

where $\mathrm{n}_{\mathrm{e}}$ is the electron density, $\overline{\mathrm{c}}_{\mathrm{e}}$ is the mean thermal velocity of electrons, and $\sigma_{\mathrm{jC}, \mathrm{ic}}$ is the effective cross-section of collisions, computed using data from Rejoub et al., Bell et al., and Borovik.[insert citation] Charge exchange rates equation shown in Eq. (16).

$\dot{\mathrm{n}}_{\mathrm{CEX}, \mathrm{iC}, \mathrm{iF}}=\mathrm{n}_{\mathrm{n}} \mathrm{n}_{\mathrm{iC}, \mathrm{iF}} \mathrm{u}_{\mathrm{iC}, \mathrm{iF}, \mathrm{n}} \sigma_{\mathrm{CEX}, \mathrm{iC}, \mathrm{iF}}$ 
where $\mathrm{n}_{\mathrm{n}}$ is the neutral density, $\mathrm{u}_{\mathrm{iC}, \mathrm{iF}, \mathrm{n}}$ is the relative drift velocity between neutrals and ions of species $\mathrm{iC}, \mathrm{iF}$, and $\sigma_{\mathrm{CEX}, \mathrm{iC}, \mathrm{F}}$ is the effective cross section. These expressions are discretized employing an Eulerian, finite-volume, cellcentered algorithm with implicit time-stepping over the whole computational domain. This last feature enables substantial savings in computational cost as time-steps can be increased beyond the limits imposed by numerical Courant conditions. Quasi-neutrality is assumed in the plume, which allows for computing the plasma density directly once the densities of all ion species are known via Eq. (17).

$$
\mathrm{n}_{\mathrm{e}}=\sum_{\mathrm{iF}} \sum_{\mathrm{iC}} \mathrm{iCn}_{\mathrm{iC}, \mathrm{iF}}
$$

Note that this assumption may fail in the proximity of spacecraft surfaces due to the presence of sheaths.

Electron temperature and plasma potential are required to fully determine the properties of the plasma. In HallPlume2D, a simple diffusion energy equation, shown in Eq. (18), is solved in order to propagate the Hall2De solution for the electron temperature for the far-field.

$$
\frac{3}{2} \mathrm{q}_{\mathrm{e}} \mathrm{n}_{\mathrm{e}} \frac{\partial \mathrm{T}_{\mathrm{e}}}{\partial \mathrm{t}}=\nabla \cdot\left(\kappa_{\mathrm{e}} \nabla \mathrm{T}_{\mathrm{e}}\right)
$$

where $T_{e}$ is the electron temperature, $\mathrm{q}_{\mathrm{e}}$ is the absolute value of the electron charge in Coulombs, and $\kappa_{\mathrm{e}}$ is the thermal diffusivity.

The motion of charged particles in the far-field is subject to the presence of an electric field. Assuming that electron and ion currents are very small and comparable due to the first being used to neutralize the second, Ohm's law can be simplified as shown in Eq. (19).

$$
\mathrm{E}=-\nabla \phi=-\frac{\nabla\left(\mathrm{n}_{\mathrm{e}} \mathrm{T}_{\mathrm{e}}\right)}{\mathrm{n}_{\mathrm{e}}}
$$

Finally, the motion of neutral atoms can be modeled with free molecular flow as the typical mean free path is in the order of hundreds of meters for neutral densities on the order of $10^{18}$ particles per cubic meter and temperatures of approximately $700 \mathrm{~K}$ (typical thruster wall temperature). Therefore collisions between atoms are extremely scarce and particles can be considered to emanate in straight paths. In HallPlume2D, the flux of neutrals across the overlap region with Hall2De is computed and propagated assuming that neutrals follow straight paths. We also include the possibility of modeling background neutrals, which emanate isotropically from the boundaries of the computational domain. The effect of electron-impact ionization of atoms is considered in the neutral solver with neutral density being reduced by the ionization rate.

Figure 36 shows a comparison of the current density measured by the Faraday probe and reported by the plume model at $1 \mathrm{~m}$ away from the HERMeS TDU1 operating at 600-12.5 throttle point. The Faraday probe result was extrapolated to zero pressure in order to provide a better comparison to the plume model results. This instance of the plume model used two ion fluids (one fast and one slow) and three charged species per fluid for a total of six individual ion populations. The travel direction and magnitude of each ion population was calculated at the locations where probing took place. Cosine of the difference between the probe angle and the travel angle was applied to obtain the current density that would have been seen by the Faraday probe. The six components of the ion current density were then summed up.

From Fig. 36, one can see that the overall shape of the measured ion current density profile and the plume model prediction matches well. Specific regions of the plume such

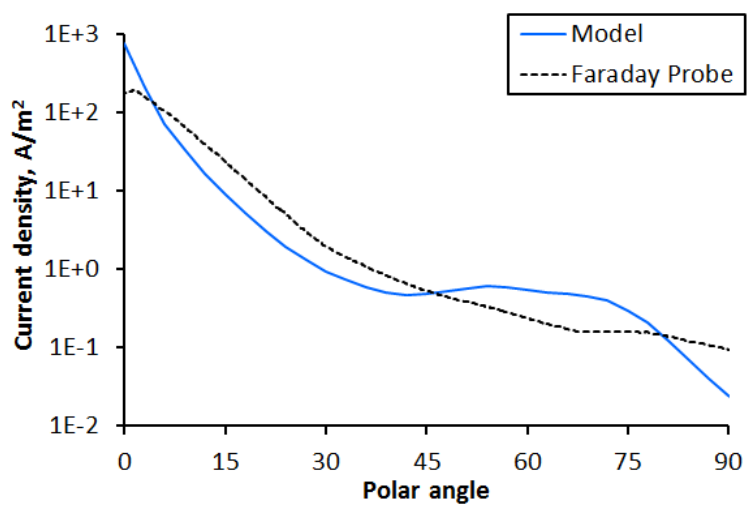

Figure 36. Comparison of current density calculated from Faraday probe data and the plume model for the 600-12.5 throttle point at $1 \mathrm{~m}$ away from the HERMeS TDU1.

as the wing can still use additional fine tuning. The team is actively pursuing the use of laser-based diagnostics to obtain data from the interior of the discharge channel in order to further fine tune inputs to Hall2De. The refined output from Hall2De should provide further improvement in the accuracy of the HallPlume2D predictions. 


\section{Conclusions}

Addressing the first primary objective of the FECT, the test results reported in this paper successfully demonstrated that the tools being used to predict zero-pressure thruster and plume characteristics are self-consistent. The test results revealed the background pressure under which the tools remain self-consistent. A wide variety of thruster and plume characteristics were used to establish criterion on maximum background pressure for testing the HERMeS TDU. In particular, ion current density, thrust, and efficiency data all pointed to a limit of approximately 1e-5 Torr background pressure in the vicinity of the thruster. The more the background pressure rose past this limit the more nonlinear the measured characteristics became. Ion energy per charge of the main beam and multiplycharged species fractions were much more tolerant to rising background pressure and did not present an important limit on the background pressure. One can conclude that from the perspective of performance analysis, data should be obtained at multiple background pressures below 1e-5 Torr and extrapolate linearly to obtain zero pressure behavior. Alternately, if a background pressure of $4 \mathrm{e}-6$ Torr is achieved, the data taken may be sufficiently close to zero pressure behavior.

Oscillation magnitude did not vary meaningfully with background pressure though frequencies did change noticeably for some operating conditions. For the purpose of discharge electrical filter design, testing below 1e-5 Torr will provide sufficiently accurate results as long as appropriate margin for the oscillation frequency is planned.

Thus, for the purpose of the first primary objective of the FECT, the team has determined that the tools for predicting zero-pressure behavior will yield self-consistent results as long as testing is performed at multiple background pressures below 1e-5 Torr.

Addressing the second primary objective of the FECT, which was to supply plasma data for spacecraft interaction studies, the team discovered a background pressure as low as 1e-6 Torr may be necessary to achieve $10 \%$ measurement accuracy. Such a low background pressure is currently impractical to achieve and we recommend obtaining data at multiple background pressure below 1e-5 Torr, where the TDU plume behaves linearly, and use the data to calibrate a physics-based plume model. Successful comparison between the FECT data and the plume model demonstrated that the second primary objective has been achieved.

In continuing the spacecraft interaction study past the FECT, the team used the ion current density and ion energy per charge measurements to refine a physics-based plume model, called HallPlume2D. The resulting prediction from the plume model was in general agreement with the data. The team is actively pursuing the use of laser-based diagnostics to improve the inputs to Hall2De, which provides inputs to HallPlume2D. This future activity is expected to further improve the agreement between the plume model and the measurements. 


\section{Appendix}

Figures 37 to 44 show the zero-pressure current density data set obtained from FP analysis.

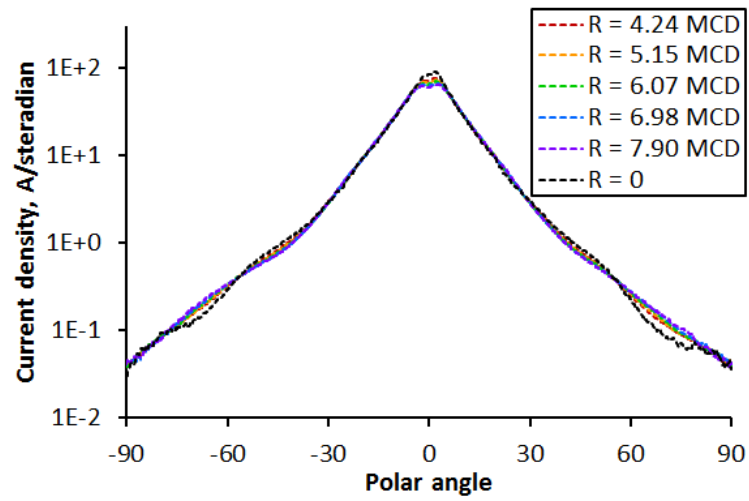

Figure 37. Extrapolated zero-pressure current density profiles at varying distances for the 300-4.7 throttle point.

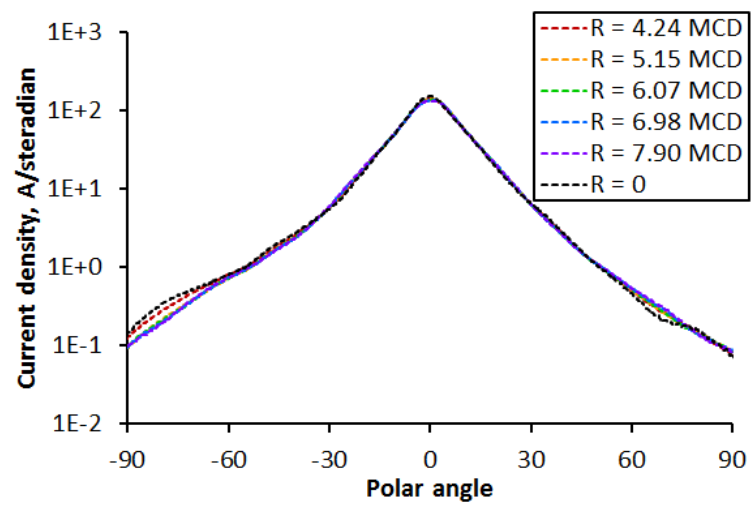

Figure 39. Extrapolated zero-pressure current density profiles at varying distances for the 400-12.5 throttle point.

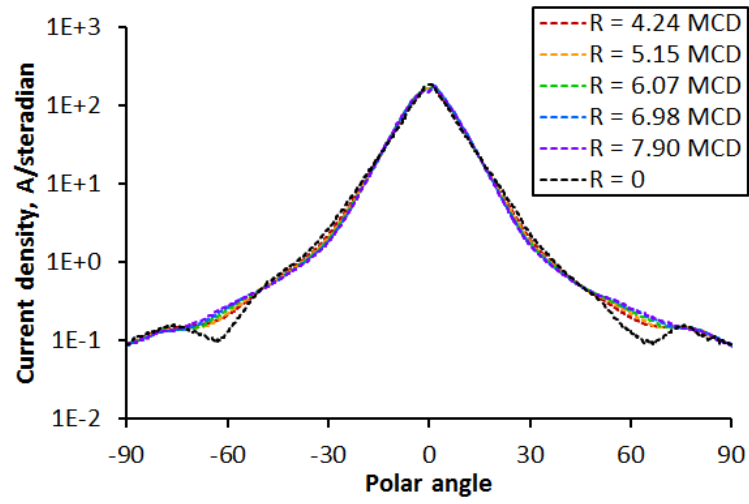

Figure 41. Extrapolated zero-pressure current density profiles at varying distances for the 600-12.5 throttle point.

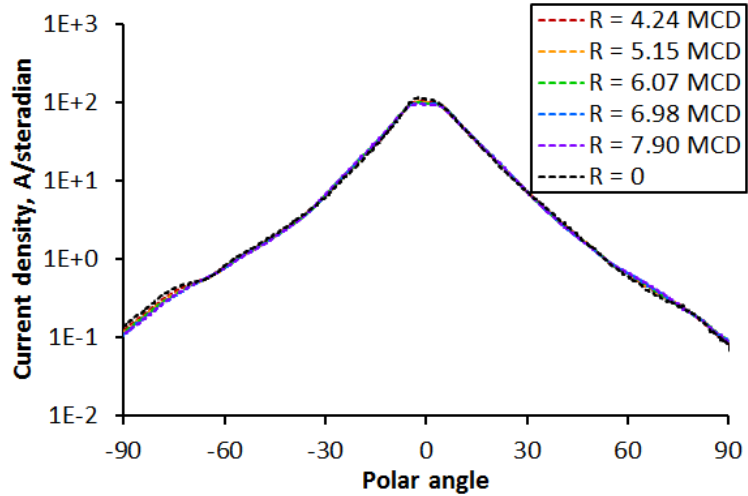

Figure 38. Extrapolated zero-pressure current density profiles at varying distances for the 300-9.4 throttle point.

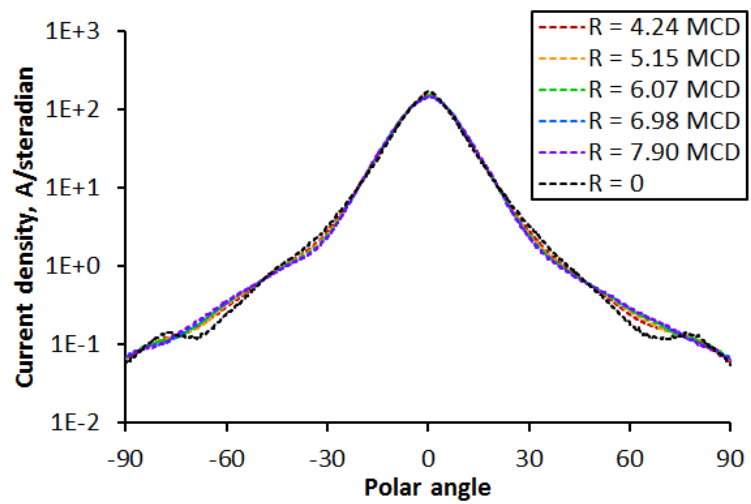

Figure 40. Extrapolated zero-pressure current density profiles at varying distances for the 500-12.5 throttle point.

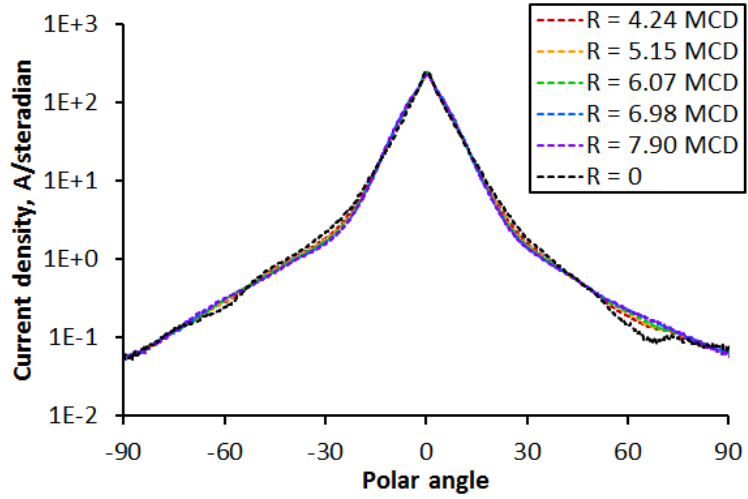

Figure 42. Extrapolated zero-pressure current density profiles at varying distances for the 700-12.5 throttle point. 


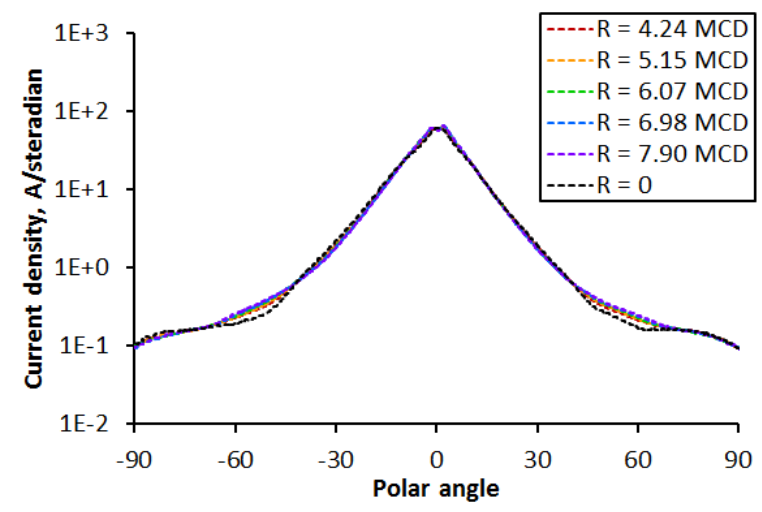

Figure 43. Extrapolated zero-pressure current density profiles at varying distances for the 800-9.7 throttle point.

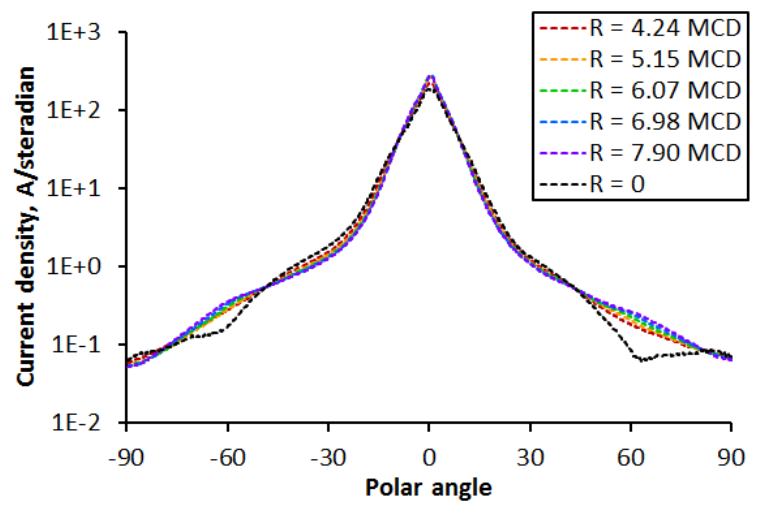

Figure 44. Extrapolated zero-pressure current density profiles at varying distances for the 800-12.5 throttle point.

Figures 45 to 52 show the contour plots of the PDFs of the thruster light intensity as the background pressure varied.

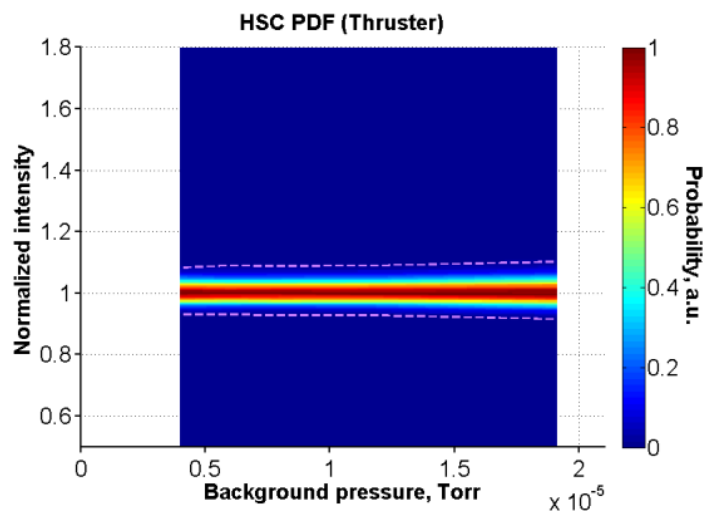

Figure 45. Contour plot of the PDF of the thruster light intensity as background pressure varied for the 300-4.7 throttle point.

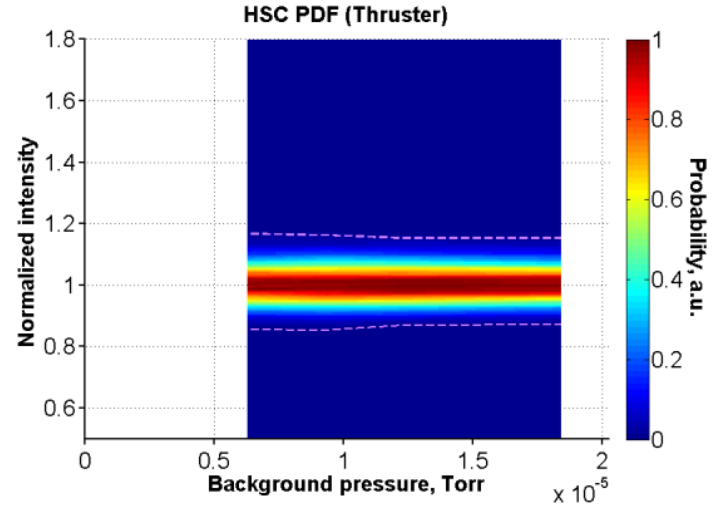

Figure 47. Contour plot of the PDF of the thruster light intensity as background pressure varied for the 400-12.5 throttle point.

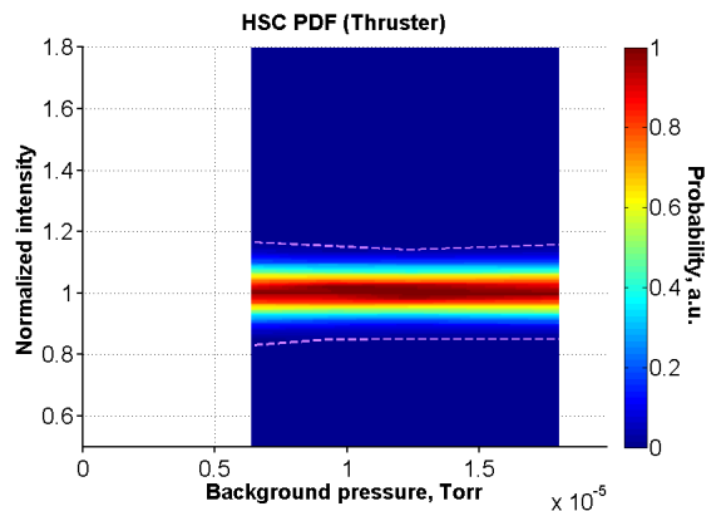

Figure 46. Contour plot of the PDF of the thruster light intensity as background pressure varied for the 300-9.4 throttle point.

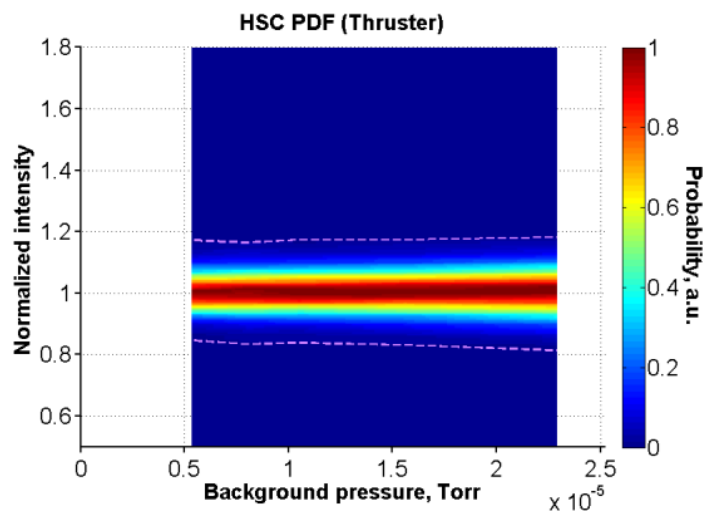

Figure 48. Contour plot of the PDF of the thruster light intensity as background pressure varied for the 500-12.5 throttle point. 


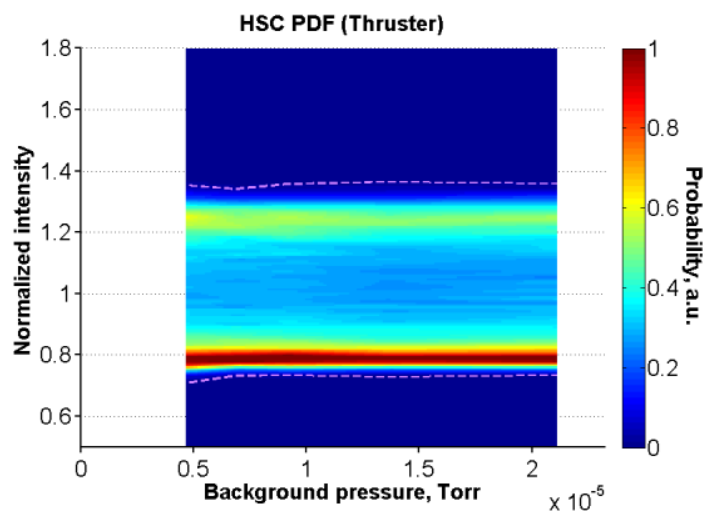

Figure 49. Contour plot of the PDF of the thruster light intensity as background pressure varied for the 600-12.5 throttle point.

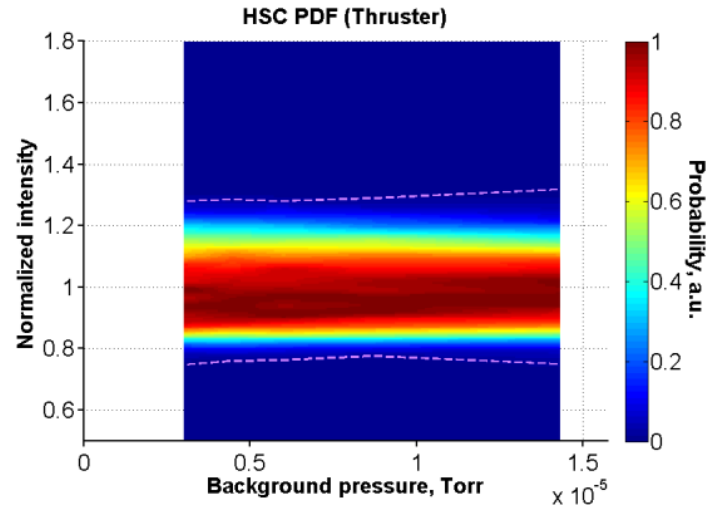

Figure 51. Contour plot of the PDF of the thruster light intensity as background pressure varied for the 800-9.7 throttle point.

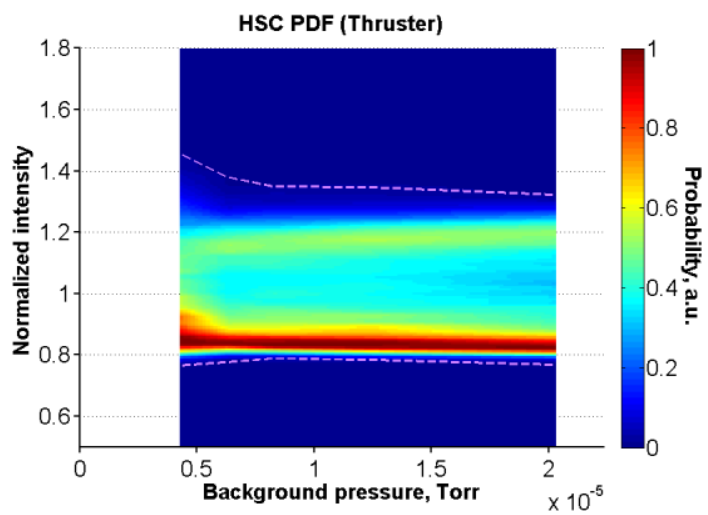

Figure 50. Contour plot of the PDF of the thruster light intensity as background pressure varied for the 700-12.5 throttle point.

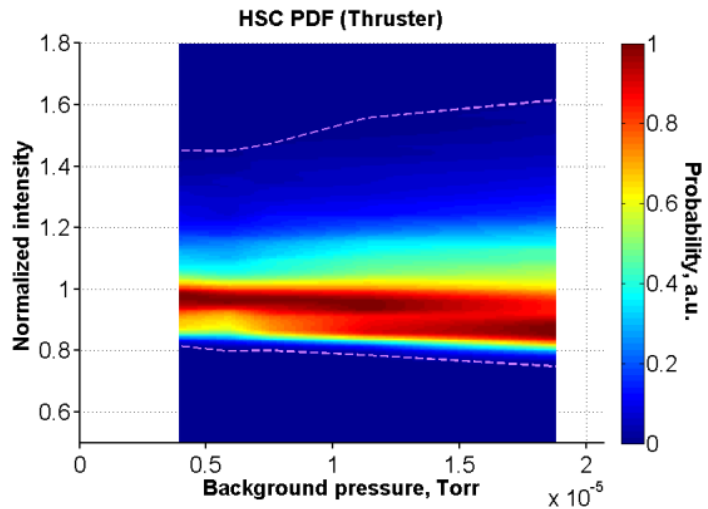

Figure 52. Contour plot of the PDF of the thruster light intensity as background pressure varied for the 800-12.5 throttle point.

Figures 53 to 60 show the contour plots of the $m=0$ power spectra of the thruster light intensity as the background pressure varied.

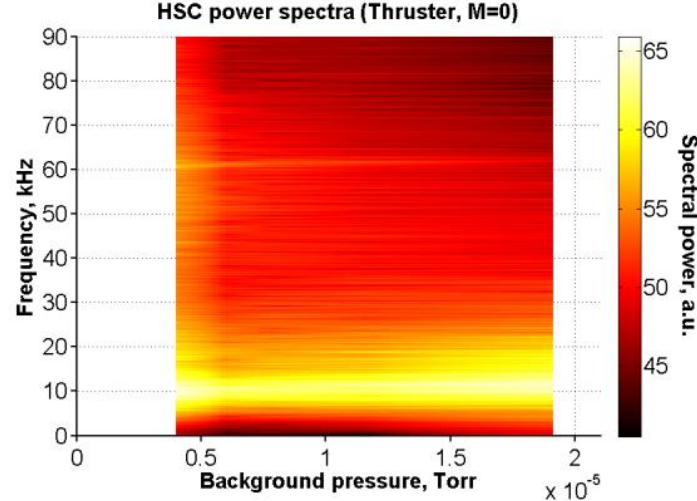

Figure 53. Contour plot of the $m=0$ power spectra of the thruster light intensity as background pressure varied for the 300-4.7 throttle point.

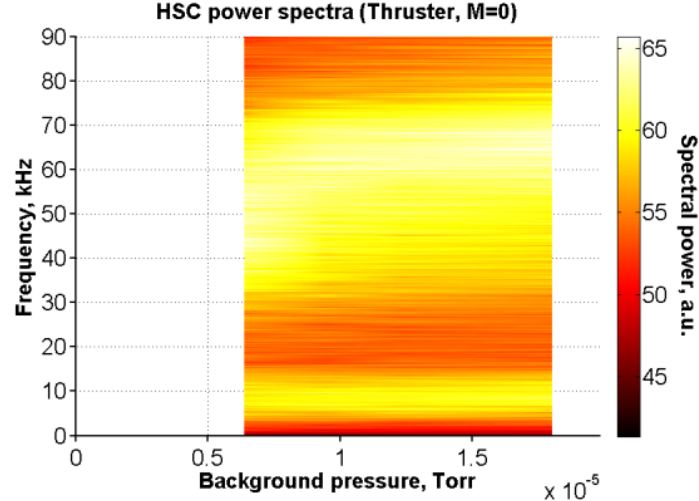

Figure 54. Contour plot of the $m=0$ power spectra of the thruster light intensity as background pressure varied for the 300-9.4 throttle point. 


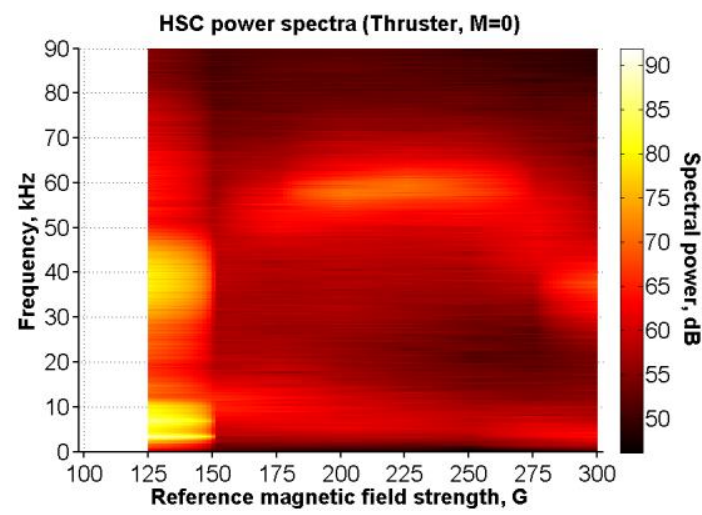

Figure 55. Contour plot of the $\mathbf{m}=\mathbf{0}$ power spectra of the thruster light intensity as background pressure varied for the $400-12.5$ throttle point.

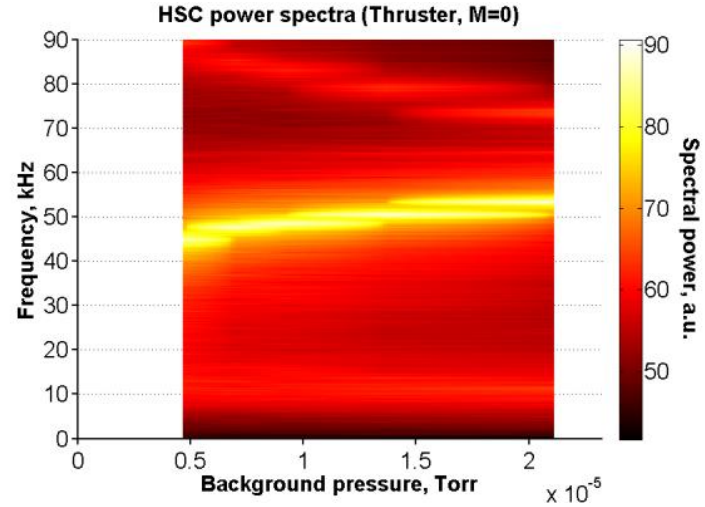

Figure 57. Contour plot of the $\mathbf{m}=\mathbf{0}$ power spectra of the thruster light intensity as background pressure varied for the 600-12.5 throttle point.

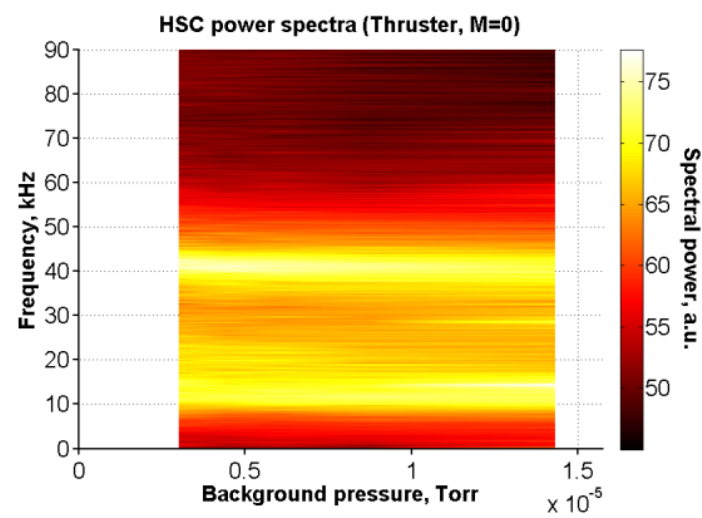

Figure 59. Contour plot of the $\mathbf{m}=\mathbf{0}$ power spectra of the thruster light intensity as background pressure varied for the 800-9.7 throttle point.

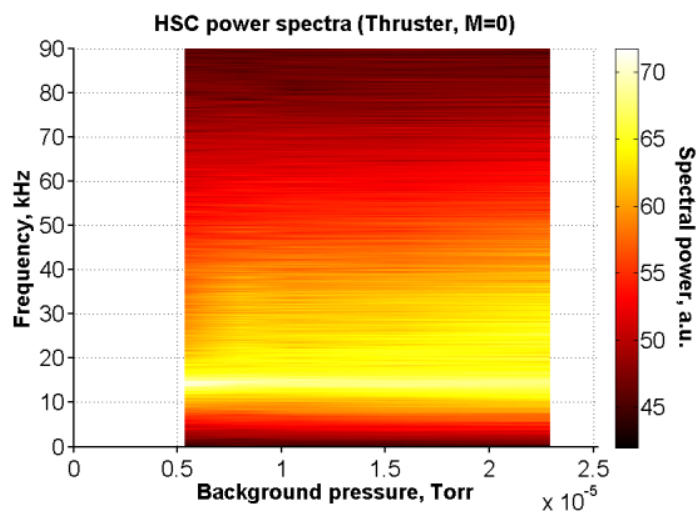

Figure 56. Contour plot of the $\mathrm{m}=0$ power spectra of the thruster light intensity as background pressure varied for the 500-12.5 throttle point.

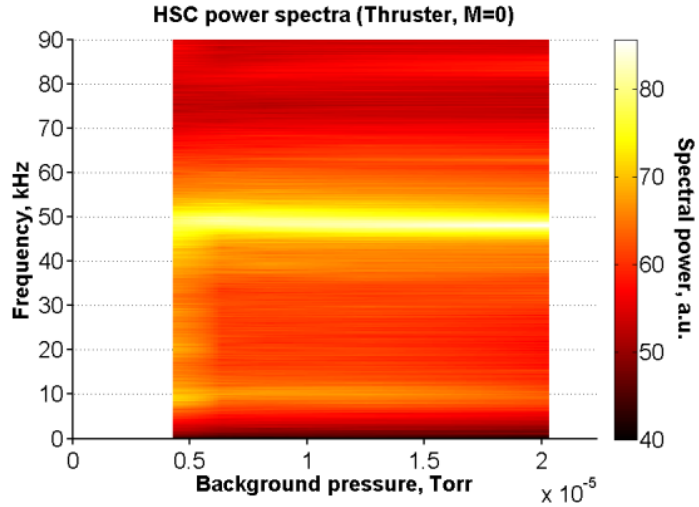

Figure 58. Contour plot of the $m=0$ power spectra of the thruster light intensity as background pressure varied for the 700-12.5 throttle point.

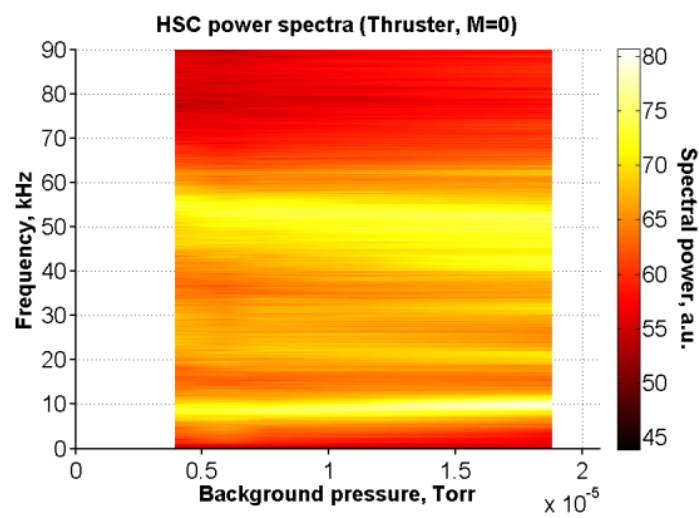

Figure 60. Contour plot of the $m=0$ power spectra of the thruster light intensity as background pressure varied for the $800-12.5$ throttle point.

Figures 61 to 68 show the contour plots of the $m=1$ power spectra of the cathode light intensity as the background pressure varied. 


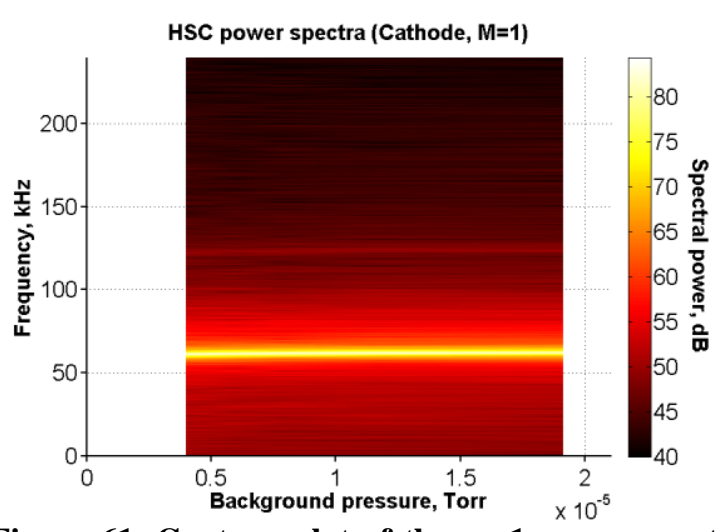

Figure 61. Contour plot of the $m=1$ power spectra of the cathode light intensity as background pressure varied for the 300-4.7 throttle point.

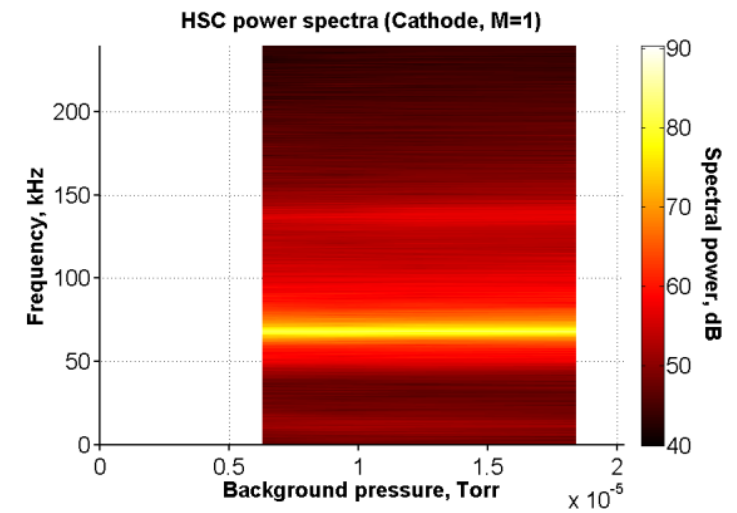

Figure 63. Contour plot of the $m=1$ power spectra of the cathode light intensity as background pressure varied for the 400-12.5 throttle point.

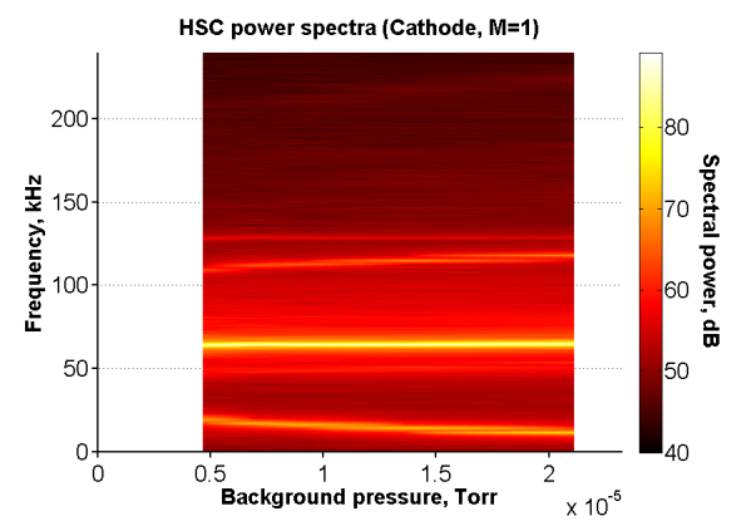

Figure 65. Contour plot of the $m=1$ power spectra of the cathode light intensity as background pressure varied for the $600-12.5$ throttle point.

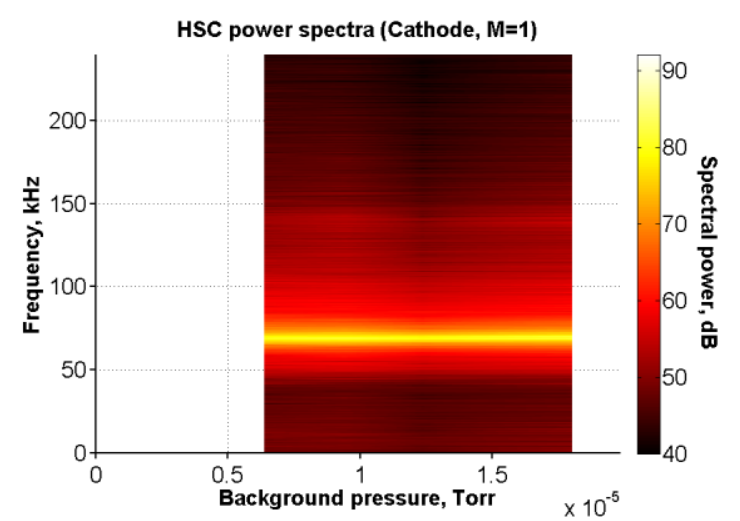

Figure 62. Contour plot of the $m=1$ power spectra of the cathode light intensity as background pressure varied for the 300-9.4 throttle point.

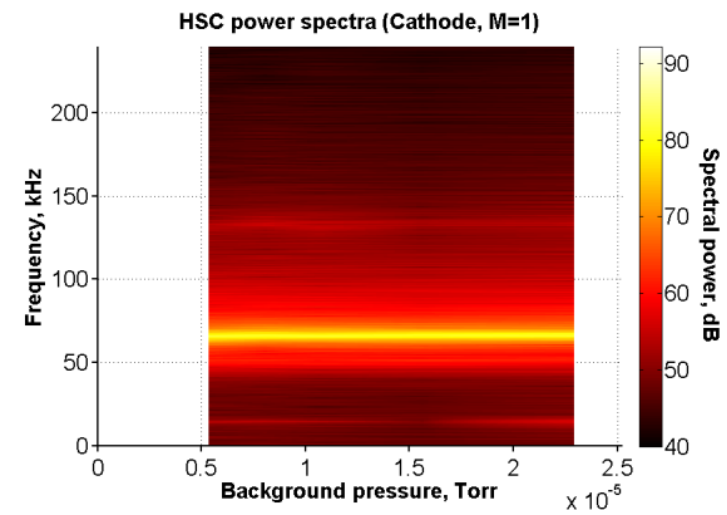

Figure 64. Contour plot of the $m=1$ power spectra of the cathode light intensity as background pressure varied for the 500-12.5 throttle point.

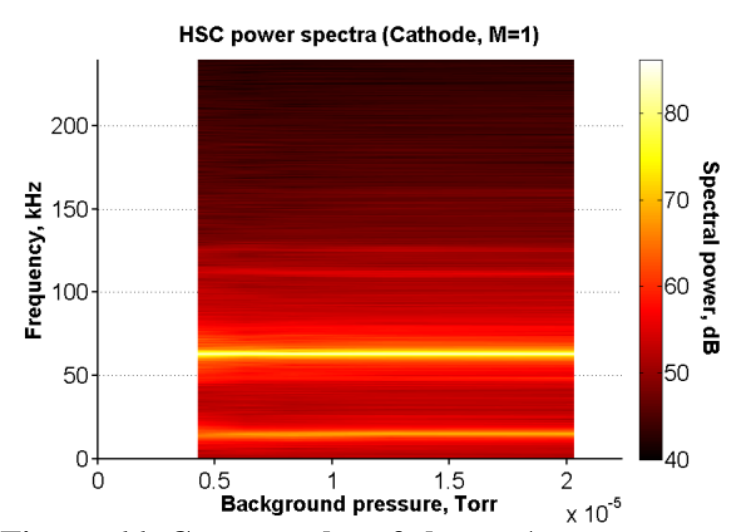

Figure 66. Contour plot of the $m=1$ power spectra of the cathode light intensity as background pressure varied for the 700-12.5 throttle point. 


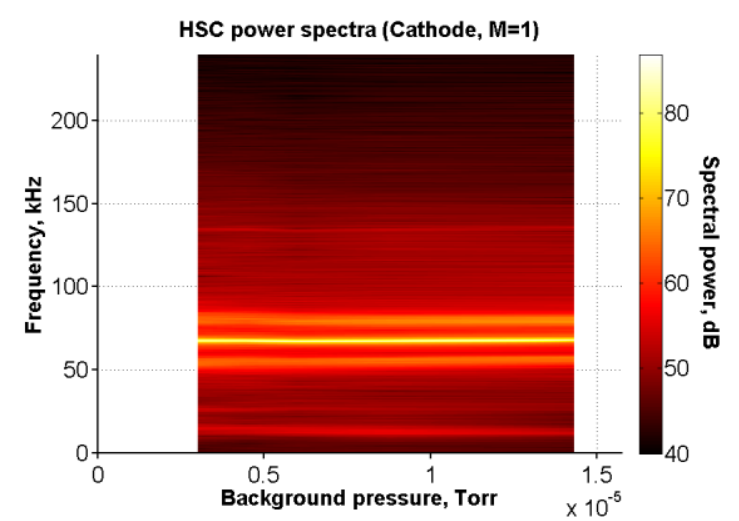

Figure 67. Contour plot of the $m=1$ power spectra of the cathode light intensity as background pressure varied for the 800-9.7 throttle point.

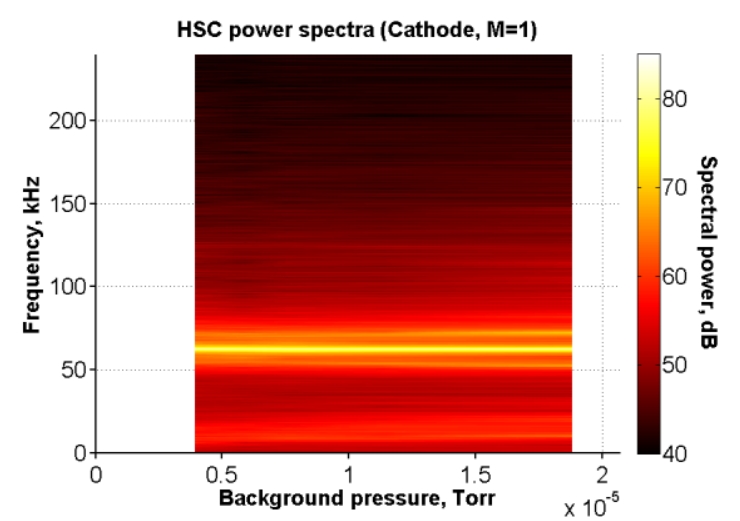

Figure 68. Contour plot of the $m=1$ power spectra of the cathode light intensity as background pressure varied for the $800-12.5$ throttle point.

Figures 69 to 71 show additional plots of ion energy per charge distribution at various background pressures for different throttle points. The polar angle of the plot shown was picked based on the largest angle at which beam ions were detected.

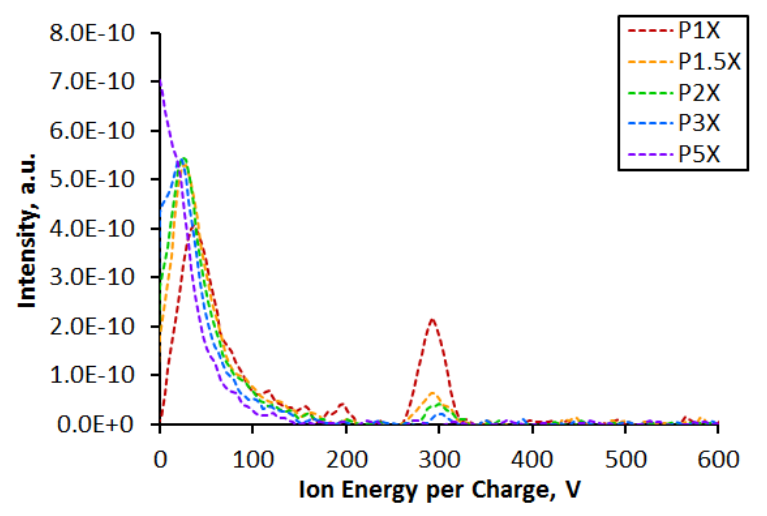

Figure 69. Ion energy per charge profiles at various background pressures for the 300-4.7 throttle point at a polar angle of $-90^{\circ}$.

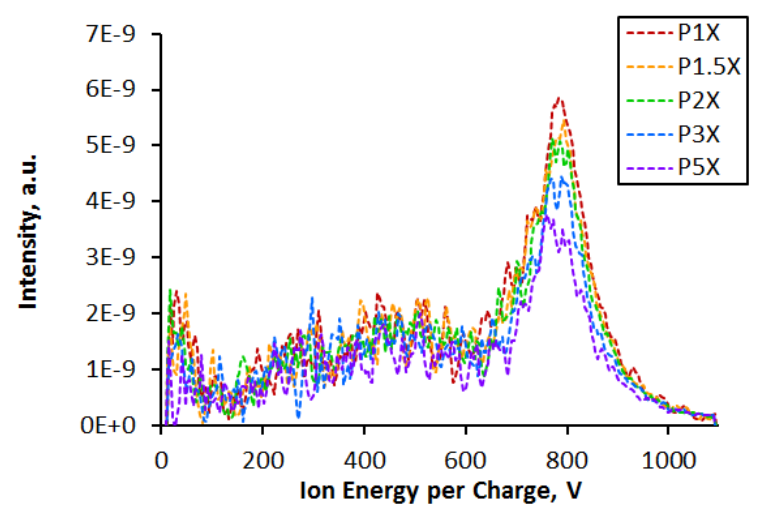

Figure 71. Ion energy per charge profiles at various background pressures for the 800-9.7 throttle point at a polar angle of $\mathbf{- 3 0}$.

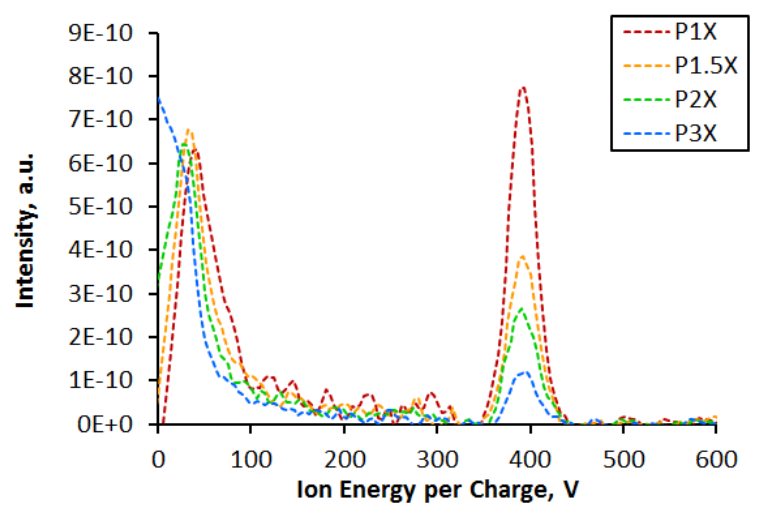

Figure 70. Ion energy per charge profiles at various background pressures for the 400-12.5 throttle point at a polar angle of $\mathbf{- 9 0 ^ { \circ }}$. 


\section{Acknowledgments}

The authors would like to thank the Space Technology Mission Directorate through the Solar Electric Propulsion Technology Demonstration Mission Project for funding the joint NASA GRC and JPL development of the HERMeS thruster and this work. We thank the Ion Propulsion Subsystem Lead, Daniel A. Herman, and the thruster lead, Richard R. Hofer, for leading this effort. We thank Christopher M. Griffiths, John T. Yim, Lauren K. Clayman, James L. Myers, Li C. Chang, Dale A. Robinson, Peter Peterson, George Williams, James Gilland of the NASA Glenn Research Center and Benjamin Jorns, James E. Polk, Michael J. Sekerak, Ryan Conversano of the Jet Propulsion Laboratory for work on the SEP TDM HERMeS Hall thruster. And we thank Kevin L. Blake, George P. Jacynycz, Thomas A. Ralys, and Terrell J. Jensen for the fabrication, assembly of the test setup, and operation of the vacuum facility. A portion of this research was carried out at the Jet Propulsion Laboratory, California Institute of Technology, under a contract with the National Aeronautics and Space Administration.

\section{References}

${ }^{1}$ Smith, B. K., Nazario, M. L., and Cunningham, C. C., "Solar Electric Propulsion Vehicle Demonstration to Support Future Space Exploration Missions", Space Propulsion 2012, Bordeaux, France, May 7-10, 2012.

${ }^{2}$ Manzella, D. and Hack, K., "High-Power Solar Electric Propulsion for Future NASA Missions", 50th AIAA/ASME/SAE/ASEE Joint Propulsion Conference, AIAA-2014-3718, doi:10.2514/6.2014-3718, Cleveland, OH, Jul 28-30, 2014.

${ }^{3}$ Kamhawi, H., et al., "Overview of the Development of the Solar Electric Propulsion Technology Demonstration Mission 12.5kW Hall Thruster", 50th AIAA/ASME/SAE/ASEE Joint Propulsion Conference, AIAA-2014-3898, doi:10.2514/6.2014-3898, Cleveland, OH, Jul 28-30, 2014.

${ }^{4}$ Brophy, J. R. and Muirhead, B., "Near-Earth Asteroid Retrieval Mission (ARM) Study", 33rd International Electric Propulsion Conference, 2013-082, Washington, DC, Oct 6-10, 2013.

${ }^{5}$ Muirhead, B. K. and Brophy, J. R., "Asteroid Redirect Robotic Mission Feasibility Study", 2014 IEEE Aerospace Conference, doi:10.1109/AERO.2014.6836358, Big Sky, MT, Mar 1-8, 2014.

${ }^{6}$ Gates, M., et al., "NASA's Asteroid Redirect Mission Concept Development Summary", 2015 IEEE Aerospace Conference, doi:10.1109/AERO.2015.7119163, Big Sky, MT, Mar 7-14, 2015.

${ }^{7}$ Mazanek, D. D., et al., "Asteroid Redirect Robotic Mission: Robotic Boulder Capture Option Overview", AIAA SPACE 2014 Conference and Exposition, AIAA-2014-4432, doi:10.2514/6.2014-4432, San Diego, CA, Aug 4-7, 2014.

${ }^{8}$ Huang, W., Yim, J. T., and Kamhawi, H., "Design and Empirical Assessment of the HERMeS Hall Thruster Propellant Manifold", 62nd Joint Army-Navy-NASA-Air Force Propulsion Meeting, JANNAF-2015-3926, Nashville, TN, Jun 1-4, 2015.

${ }^{9}$ Shastry, R., Huang, W., and Kamhawi, H., "Near-Surface Plasma Characterization of the 12.5-kW NASA TDU1 Hall Thruster", 51st AIAA/ASME/SAE/ASEE Joint Propulsion Conference, AIAA-2015-3919, doi:10.2514/6.2015-3919, Orlando, FL, Jul 27-29, 2015.

${ }^{10}$ Kamhawi, H., et al., "Performance and Facility Background Pressure Characterization Tests of NASA's 12.5-kW Hall Effect Rocket with Magnetic Shielding Thruster", 34th International Electric Propulsion Conference, 2015-007, Kobe, Japan, Jul 4-10, 2015.

${ }^{11}$ Huang, W., Kamhawi, H., Myers, J. L., Yim, J. T., and Neff, G., "Non-Contact Thermal Characterization of NASA's HERMeS Hall Thruster", 51 st AIAA/ASME/SAE/ASEE Joint Propulsion Conference, AIAA-2015-3920, doi:10.2514/6.2015-3920, Orlando, FL, Jul 27-29, 2015.

${ }^{12}$ Peterson, P. Y., et al., "NASA's HERMeS Hall Thruster Electrical Configuration Characterization ", 52nd AIAA/ASME/SAE/ASEE Joint Propulsion Conference, Salt Lake City, UT, Jul 25-27, 2016.

${ }^{13}$ Huang, W., Kamhawi, H., Lobbia, R. B., and Brown, D. L., "Effect of Background Pressure on the Plasma Oscillation Characteristics of the HiVHAc Hall Thruster", 50th AIAA/ASME/SAE/ASEE Joint Propulsion Conference, AIAA-2014-3708, doi:10.2514/6.2014-3708, Cleveland, OH, Jul 28-30, 2014.

${ }^{14}$ Hofer, R. R. and Anderson, J. R., "Finite Pressure Effects in Magnetically Shielded Hall Thrusters", 50th AIAA/ASME/SAE/ASEE Joint Propulsion Conference, AIAA-2014-3709, doi:10.2514/6.2014-3709, Cleveland, OH, Jul 28-30, 2014.

${ }^{15}$ Diamant, K. D., Liang, R., and Corey, R. L., "The Effect of Background Pressure on SPT-100 Hall Thruster Performance", 50th AIAA/ASME/SAE/ASEE Joint Propulsion Conference, AIAA-2014-3710, doi:10.2514/6.2014-3710, Cleveland, OH, Jul 28-30, 2014.

${ }^{16}$ Gilland, J. H., Williams, G. J., Burt, J. M., and Yim, J. T., "Carbon Back Sputter Modeling for Hall Thruster Testing", 52nd AIAA/ASME/SAE/ASEE Joint Propulsion Conference, Salt Lake City, UT, Jul 25-27, 2016.

${ }^{17}$ Yim, J. T. and Burt, J. M., "Characterization of Vacuum Facility Background Gas Through Simulation and Considerations for Electric Propulsion Ground Testing", 51st AIAA/ASME/SAE/ASEE Joint Propulsion Conference, AIAA-2015-3825, doi:10.2514/6.2015-3825, Orlando, FL, Jul 27-29, 2015.

${ }^{18} \mathrm{Haag}, \mathrm{T}$. W., "Thrust stand for high-power electric propulsion devices", Review of Scientific Instruments, Vol. 62, No. 5, doi:10.1063/1.1141998, May, 1991, pp. 1186.

${ }^{19}$ Huang, W., Shastry, R., Soulas, G. C., and Kamhawi, H., "Farfield Plume Measurement and Analysis on the NASA-300M and NASA-300MS", 33rd International Electric Propulsion Conference, 2013-057, Washington, DC, Oct 6-10, 2013. 
${ }^{20}$ Huang, W., Kamhawi, H., and Haag, T., "Effect of Background Pressure on the Performance and Plume of the HiVHAc Hall Thruster", 33rd International Electric Propulsion Conference, 2013-058, Washington, DC, Oct 6-10, 2013.

${ }^{21}$ Brown, D. L., "Investigation of Flow Discharge Voltage Hall Thruster Characteristics and Evaluation of Loss Mechanisms", Ph.D. Dissertation, Aerospace Engineering, University of Michigan, Ann Arbor, MI, 2009.

${ }^{22}$ Shastry, R., Hofer, R. R., Reid, B. M., and Gallimore, A. D., "Method for analyzing ExB probe spectra from Hall thruster plumes", Review of Scientific Instruments, Vol. 80, No. 6, doi:10.1063/1.3152218, Jun 22, 2009, pp. 063502.

${ }^{23} \mathrm{Kim}, \mathrm{V}$., "Main physical features and processes determining the performance of stationary plasma thrusters", Journal of Propulsion and Power, Vol. 14, No. 5, 1998, pp. 736-743.

${ }^{24}$ Hofer, R. R. and Gallimore, A. D., "High-Specific Impulse Hall Thrusters, Part 2: Efficiency Analysis", Journal of Propulsion and Power, Vol. 22, No. 4, doi:10.2514/1.15954, Jul-Aug, 2006, pp. 732-740.

${ }^{25}$ Reid, B. M., "The Influence of Neutral Flow Rate in the Operation of Hall Thrusters", Ph.D. Dissertation, Aerospace Engineering, University of Michigan, Ann Arbor, MI, 2008.

${ }^{26}$ Brown, D. L. and Gallimore, A. D., "Evaluation of Ion Collection Area in Faraday probes", Review of Scientific Instruments, Vol. 81, No. 6, doi:10.1063/1.3449541, Jun 25, 2010, pp. 063504.

${ }^{27}$ Brown, D. L. and Gallimore, A. D., "Evaluation of Facility Effects on Ion Migration in a Hall Thruster Plume", Journal of Propulsion and Power, Vol. 27, No. 3, doi:10.2514/1.B34068, May-Jun, 2011, pp. 573-585.

${ }^{28}$ Hagstrum, H. D., "Auger Ejection of Electrons from Tungsten by Noble Gas Ions", Physical Review, Vol. 96, No. 2, doi:10.1103/PhysRev.96.325, Oct 15, 1954, pp. 325-335.

${ }^{29}$ Hagstrum, H. D., "Auger Ejection of Electrons from Molybdenum by Noble Gas Ions", Physical Review, Vol. 104, No. 3, doi:10.1103/PhysRev.104.672, Nov 1, 1956, pp. 672-683.

${ }^{30}$ Hagstrum, H. D., "Auger Ejection of Electrons from Tungsten by Noble Gas Ions", Physical Review, Vol. 104, No. 2, doi:10.1103/PhysRev.104.317, Oct 15, 1956, pp. 317-318.

${ }^{31}$ Hagstrum, H. D., "Metastable Ions of the Noble Gases", Physical Review, Vol. 104, No. 2, doi:10.1103/PhysRev.104.309, Sep, 1956, pp. 309-316.

${ }^{32}$ Lieberman, M. A. and Lichtenberg, A. J., Electrostatic Probe Diagnostics, in Principles of Plasma Discharges and Materials Processing, 2nd ed., Ch., doi:10.1002/0471724254, Wiley, New York, 2005, pp. 185-203.

${ }^{33}$ Savitzky, A. and Golay, M. J. E., "Smoothing and Differentiation of Data by Simplified Least Squares Procedures", Analytical Chemistry, Vol. 36, No. 8, doi:10.1021/ac60214a047, Jul, 1964, pp. 1627-1639.

${ }^{34}$ Steinier, J., Termonia, Y., and Deltour, J., "Comments on Smoothing and Differentiation of Data by Simplified Least Squares Procedure", Analytical Chemistry, Vol. 44, No. 11, doi:10.1021/ac60319a045, Sep, 1972, pp. 1906-1909.

${ }^{35}$ Hutchinson, I. H., Principles of Plasma Diagnostics, 2nd ed., doi:10.1017/CBO9780511613630, Cambridge University Press, 2002.

${ }^{36}$ Huang, W. and Shastry, R., "Analysis of Wien filter spectra from Hall thruster plumes", Review of Scientific Instruments, Vol. 86, No. 7, doi:10.1063/1.4923282, Jul 6, 2015, pp. 073502.

${ }^{37}$ Jorns, B. A. and Hofer, R. R., "Plasma oscillations in a 6-kW magnetically shielded Hall thruster", Physics of Plasmas, Vol. 21, No. 5, doi:10.1063/1.4879819, May 30, 2014, pp. 053512.

${ }^{38}$ Huang, W., Kamhawi, H., and Haag, T. W., "Plasma Oscillation Characterization of NASA's HERMeS Hall Thruster via High Speed Imaging", 52nd AIAA/ASME/SAE/ASEE Joint Propulsion Conference, Salt Lake City, UT, Jul 25-27, 2016.

${ }^{39}$ Sekerak, M. J., et al., "Mode Transitions in Magnetically Shielded Hall Effect Thrusters", 50th AIAA/ASME/SAE/ASEE Joint Propulsion Conference, AIAA-2014-3511, doi:10.2514/6.2014-3511, Cleveland, OH, Jul 28-30, 2014.

${ }^{40}$ Hara, K., Sekerak, M. J., Boyd, I. D., and Gallimore, A. D., "Mode transition of a Hall thruster discharge plasma", Journal of Applied Physics, Vol. 115, No. 20, doi:10.1063/1.4879896, May 29, 2014, pp. 203304.

${ }^{41}$ Fife, J. M., Martinez-Sanchez, M., and Szabo, J., "A Numerical Study of Low-Frequency Discharge Oscillations in Hall Thrusters", 33rd AIAA/ASME/SAE/ASEE Joint Propulsion Conference, AIAA-1997-3052, doi:10.2514/6.1997-3052, Seattle, WA, Jul 6-9, 1997.

${ }^{42}$ Lobbia, R. B., "A Time-resolved Investigation of the Hall Thruster Breathing Mode", Ph.D. Dissertation, Aerospace Engineering, University of Michigan, Ann Arbor, MI, 2009.

${ }^{43}$ Diamant, K. D., et al., "Performance and Plume Characterization of the BHT-1500 Hall Thruster", 34th International Electric Propulsion Conference, 2015-069, Kobe, Japan, Jul 4-10, 2015.

${ }^{44}$ Tighe, W. G., Spektor, R., and Diamant, K. D., "Effects of Background Pressure on the NASA 173M Hall Current Thruster Performance", 34th International Electric Propulsion Conference, 2015-152, Kobe, Japan, Jul 4-10, 2015.

${ }^{45}$ Lopez Ortega, A., Katz, I., Mikellides, I. G., and Goebel, D. M., "Self-Consistent Model of a High-Power Hall Thruster Plume", IEEE Transactions on Plasma Science, Vol. 43, No. 9, doi:10.1109/TPS.2015.2446411, Sep 9, 2015, pp. $2875-2886$. 